\title{
Shielded base contraction
}

\author{
Marco Garapa $^{\mathrm{a}, \mathrm{b}, *}$, Eduardo Fermé ${ }^{\mathrm{a}, \mathrm{c}}$, Maurício D.L. Reis ${ }^{\mathrm{a}, \mathrm{b}}$ \\ a Faculdade de Ciências Exatas e da Engenharia, Universidade da Madeira, Campus Universitário da Penteada, 9020-105 Funchal, Portugal \\ b CIMA - Centro de Investigação em Matemática e Aplicações, Portugal \\ ${ }^{\mathrm{c}}$ NOVA Laboratory for Computer Science and Informatics (NOVA LINCS), Portugal
}

\section{A R T I C L E I N F O}

\section{Article history:}

Received 13 July 2017

Received in revised form 14 March 2018

Accepted 20 March 2018

Available online 23 March 2018

\section{Keywords:}

Non-prioritized belief change

Belief bases

Base contraction

Shielded contraction

Axiomatic characterizations

\begin{abstract}
A B S T R A C T
The one that is considered the standard model in the area of logic of belief change was proposed by Alchourrón, Gärdenfors and Makinson in 1985 and is, nowadays, known as the AGM model.

Two of the main shortcomings pointed out to the AGM model of belief change are the (impractical) use of belief sets to represent belief states and the (unrealistic) acceptance of any new information. In this paper we study a kind of operators-known as shielded base contractions-which address both those issues. Indeed, on the one hand, these operators are defined on belief bases (rather than belief sets) and, on the other hand, they are constructed with the underlying idea that not all new informations are accepted.

We propose twenty different classes of shielded base contractions and obtain axiomatic characterizations for each of them. Additionally we thoroughly investigate the interrelations (in the sense of inclusion) among all those classes. More precisely, we analyse whether each of those classes is or is not (strictly) contained in each of the remaining ones.
\end{abstract}

(C) 2018 Elsevier B.V. All rights reserved.

\section{Introduction}

One of the main goals underlying the research area of belief change consists in finding appropriate ways of modelling how the belief state of an (idealized rational) agent is modified when it receives some new information leading it to give up one of its current beliefs. More precisely, several of the main references of this area deal with the definition and characterization of operators that receive a set of sentences (representing the belief state of an agent) and a sentence (representing a belief that is intended to be given up by that agent) and return a set of sentences (that models the new belief state of that agent) which:

(i) is a subset of the received set of sentences;

(ii) does not logically imply the received sentence (provided that it is not a tautology).

An operator that receives a pair of inputs, as described above, and returns an output satisfying (i) and (ii) is said to satisfy the postulates of inclusion and success, respectively, and is commonly called a contraction (e.g. [24, page 65]).

The one which is currently considered the standard model in the belief change literature is known as AGM model-after the initials of its three creators: Carlos Alchourrón, Peter Gärdenfors and David Makinson-and has been originally presented in [1]. In that framework, each belief of an agent is represented by a sentence (of a propositional language $\mathcal{L}$ ) and the belief

\footnotetext{
* Corresponding author.

E-mail addresses: marco@uma.pt (M. Garapa), ferme@uma.pt (E. Fermé), m_reis@uma.pt (M.D.L. Reis).
} 
state of an agent is represented by a logically closed set of (belief-representing) sentences. These sets are called belief sets. In that paper some properties are proposed as being the characteristic properties of a contraction. Nowadays, these properties (which are recalled in Subsection 2.2) are commonly referred to as the AGM postulates for contraction and an operator that satisfies them is called an AGM contraction.

Several constructive definitions of operators which satisfy all or at least some of the AGM postulates for contraction have been provided in the literature. Some of the most well-known of those models are the (transitively relational) partial meet contractions [1], safe contraction [3,28], system of spheres-based contraction [15] and epistemic entrenchment-based contraction $[12,13]$.

Nevertheless, the AGM model has also been the subject of many critiques and, not long after its publication, several variants of that model started to appear in the literature. ${ }^{1}$ From among those proposals we highlight (for being the ones that are directly related to the present work) the following classes of belief change operators:

(a) Classes of contractions which can be seen as generalizations of (some of) the above mentioned constructive definitions of AGM contractions to the case when the belief states of an agent are represented by sets of sentences not (necessarily) closed under logical consequence-the so-called belief bases-rather than by belief sets.

(b) Classes of, so-called, non-prioritized contractions, which are "contraction" operators that do not satisfy the success postulate.

From among the classes mentioned in (a) above we highlight the partial meet contractions, presented in [19,20,17]; the kernel contractions-which can be seen as a generalization of safe contractions and are defined for belief bases-that were introduced in [21]; and the ensconcement-based contractions, that were proposed in [29] and can be seen as adaptations to the case of belief bases of the epistemic entrenchment-based contractions. These kind of change operators for belief bases have arisen in order to address the criticisms to the AGM framework concerning the use of logically closed sets (or belief sets) to model the belief state of an agent. Belief sets are very large entities, and its use is not adequate for computational implementations. Any attempt to computationally implement the theory of belief change will have to be based on a finite representation. Furthermore, as Gärdenfors and Rott pointed out "when we perform revisions or contractions, it seems that we never do it to the belief set itself (...) but rather on some typically finite base for the belief set" [14]. A set $A$ is a base for a belief set $\mathbf{K}$ if and only if $\operatorname{Cn}(A)=\mathbf{K}$. A sentence $\alpha$ is believed if and only if $\alpha \in \operatorname{Cn}(A)$.

In what concerns the classes of operators mentioned in (b), the pioneering work on that topic is [6], where Fermé and Hansson introduced the concept of shielded contraction. The motivation for the proposal of this kind of operators was the fact that, as pointed out by Rott [27], the success postulate is not a fully realistic requirement since an agent can have several (non-tautological) beliefs that he/she is not willing, for various reasons, to give up. Thus, shielded contractions are operators that for some inputs behave just as (standard) contractions and for other inputs just do not have any effect at all-in the sense that they simply return (as output) the belief state received as input.

We should, however, note here that the AGM belief change operators are not intended to force an agent to remove or incorporate beliefs that he/she is unwilling to remove or incorporate, but that such change operations are only applied for those beliefs that the agent decides to remove or incorporate after performing some type of previous processing of the information. ${ }^{2}$ In the AGM model, this information preprocessing is left implicit. In the case of the (shielded contraction) operators studied in this paper this prior information processing is made explicit by means of a specification of the set of those beliefs that the agent is willing to remove in terms of properties (whose influence in the quality of the associated shielded contraction operator is analysed).

In [6], a shielded contraction is defined by means of an AGM contraction and a set of sentences $R$ satisfying certain properties, named set of retractable sentences, which models the set of sentences that the agent is willing to give up (if needed). Informally speaking, the shielded contraction is a function that receives (just as a standard contraction does) a belief set and a sentence and returns:

- The received belief set (unchanged), if the received sentence is not included in $R$;

- The output produced by the associated AGM contraction (when it receives those two inputs), if the received sentence is in $R$.

In the present paper we define and thoroughly study twenty classes of operators, which can be thought as combining the central features of the classes of operators mentioned in (a) and (b) above, since the operators that we shall consider are "contractions" defined for belief bases and which do not (necessarily) satisfy the postulate of success. More precisely, we shall study shielded contractions defined for belief bases (rather than for belief sets). Until now, only one class of such operators has been considered in the belief change literature, namely a class of shielded contractions on belief bases defined by means of a partial meet contraction and a set of retractable sentences (satisfying certain properties), which was presented and axiomatically characterized in [10] (and which is recalled in Subsection 2.4). In this paper we consider classes

\footnotetext{
1 For an overview see [7].

2 This was made clear, for example, in [26].
} 
of shielded base contraction induced by several well-known kinds of base contractions (not only partial meet contractions) and several kinds of sets of retractable sentences (i.e. we consider several different, and non-equivalent, sets of properties for characterizing a set of retractable sentences). We axiomatically characterize all the classes of shielded base contractions considered and study the interrelations among them, namely by investigating if each of those classes is or is not (strictly) contained in each one of the remaining classes considered.

At this point we remark that the study of shielded base contractions is a relevant topic in the field of artificial intelligence with applications in different areas. For example in the database context, the programmer may specify integrity constrains that are not liable to be retracted. Another example is a normative system in which some norms are not liable to be retracted from the system. More generally shielded contractions may be useful for modelling the behaviour of an agent when confronted with some new information that contradicts one (or more) of his present beliefs. In such a situation, two reasonable possible behaviours are the following: either the agent disregards the new information (and keeps its belief state unchanged) or it accepts the new information and changes its belief state accordingly, namely by, roughly speaking, giving up the ones of its previous beliefs which are contradicted by the new information received. Indeed, it is not expectable that a rational agent will believe every new information it receives and that is the reason why shielded contraction was proposed as a two steps process. When some new information is received whose acceptance would force the removal of some belief, first it is checked whether the agent is willing to give up that belief, and only if that is confirmed to be the case, does the removal of that belief occur (in a second step). We notice that (standard) contraction functions do not model the phase of assessing the acceptability of the removal of a sentence.

The rest of the paper is organized as follows: In Section 2 we introduce the notations and recall the main background concepts that will be needed throughout this article. In Section 3 we present a formal definition of shielded base contraction and introduce some desirable properties that a given set of retractable sentences should satisfy. We present several results highlighting the interrelations among the properties satisfied by a shielded base contraction operator and the properties satisfied by the (standard) contraction and the set of retractable sentences which induce that shielded contraction. Afterwards, in Section 4 we present axiomatic characterizations for the following classes of shielded base contractions: shielded contractions induced by partial meet contractions, (two classes of) shielded contractions induced by kernel contractions, and shielded contractions induced by basic AGM-generated base contractions. For each one of these four classes, we shall identify five different subclasses-each one associated to a certain list of properties of the set of retractable sentences. In Section 5 we analyse the interrelations among (all) the classes of shielded contractions considered in terms of the relation of (strict) inclusion. In Section 6 we briefly recall the main operators of non-prioritized belief change (other than shielded contractions) so far presented in the literature. Finally, in Section 7 we summarize the main contributions of the paper and briefly discuss their relevance. In the Appendix we provide proofs for all the original results presented.

\section{Background}

\subsection{Formal preliminaries}

We will assume a propositional language $\mathcal{L}$ that contains the usual truth functional connectives: $\neg$ (negation), $\wedge$ (conjunction), $\vee$ (disjunction), $\rightarrow$ (implication) and $\leftrightarrow$ (equivalence). We shall make use of a consequence operation $C n$ that takes sets of sentences to sets of sentences and which satisfies the standard Tarskian properties, namely inclusion, monotony and iteration. Furthermore we will assume that $C n$ satisfies supraclassicality, compactness and deduction. We will sometimes use $C n(\alpha)$ for $C n(\{\alpha\}), A \vdash \alpha$ for $\alpha \in C n(A), \vdash \alpha$ for $\alpha \in C n(\emptyset), A \nvdash \alpha$ for $\alpha \notin C n(A), \nvdash \alpha$ for $\alpha \notin C n(\emptyset)$. The letters $\alpha, \beta, \ldots$ (except for $\gamma$ ) will be used to denote sentences of $\mathcal{L}$. Lowercase Latin letters such as $p, q, \ldots$ will be used to denote atomic sentences of $\mathcal{L}$. $A, B, \ldots$ shall denote sets of sentences of $\mathcal{L}$. $\mathbf{K}$ is reserved to represent a set of sentences that is closed under logical consequence (i.e. $\mathbf{K}=C n(\mathbf{K})$ )-such a set is called a belief set or theory. Given $A \subseteq \mathcal{L}$, we shall say that - is a (contraction) operator (or function) on $A$ when - is a function such that $-: \mathcal{L} \longrightarrow P(\mathcal{L})$. Furthermore, in that context, we shall represent by $A-\alpha$ the image of a sentence $\alpha$ by - .

\subsection{AGM contractions and their associated shielded contractions}

We start this subsection by recalling the AGM postulates for contraction and the concept of AGM contraction.

Definition 2.1 ([1]). Let $\mathbf{K}$ be a belief set. An operator - on $\mathbf{K}$ is an AGM contraction if and only if it satisfies the following conditions:

$$
\begin{array}{ll}
(\mathbf{K}-1) & \mathbf{K}-\alpha=C n(\mathbf{K}-\alpha) . \\
(\mathbf{K}-2) & \mathbf{K}-\alpha \subseteq \mathbf{K} . \\
(\mathbf{K}-3) & \text { If } \alpha \notin \mathbf{K} \text {, then } \mathbf{K}-\alpha=\mathbf{K} . \\
(\mathbf{K}-4) & \text { If } \alpha \notin C n(\emptyset), \text { then } \alpha \notin \mathbf{K}-\alpha . \\
(\mathbf{K}-5) & \text { If } \alpha \leftrightarrow \beta \in C n(\emptyset), \text { then } \mathbf{K}-\alpha=\mathbf{K}-\beta . \\
(\mathbf{K}-6) & \mathbf{K} \subseteq C n((\mathbf{K}-\alpha) \cup\{\alpha\}) .
\end{array}
$$


$(\mathbf{K}-7) \quad(\mathbf{K}-\alpha) \cap(\mathbf{K}-\beta) \subseteq \mathbf{K}-(\alpha \wedge \beta)$.

$(\mathbf{K}-8) \quad \mathbf{K}-(\alpha \wedge \beta) \subseteq \mathbf{K}-\alpha$ whenever $\alpha \notin \mathbf{K}-(\alpha \wedge \beta)$.

(Conjunctive overlap)

(Conjunctive inclusion)

These conditions are called the AGM postulates for contraction. Postulates $(\mathbf{K}-1)-(\mathbf{K}-6)$ are named basic AGM postulates for contraction and an operator - that satisfies those properties is called a basic AGM contraction. Postulates ( $\mathbf{K}-7)$ and $(\mathbf{K}-8)$ are designated by supplementary AGM postulates for contraction.

Motivated by the fact that an agent can have several non-tautological beliefs that he/she is not willing to give up, Fermé and Hansson [6] presented a new kind of operators, called shielded contractions, constructed by means of a basic AGM contraction and a set of sentences $R$, as we recall in the following definition.

Definition 2.2 ([6]). Let $\mathbf{K}$ be a belief set, - be a basic AGM contraction on $\mathbf{K}$ and $R$ a subset of $\mathcal{L}$. Then $\rightarrow$ is the shielded contraction operator induced by - and $R$ if and only if:

$$
\mathbf{K} \rightarrow \alpha= \begin{cases}\mathbf{K}-\alpha & \text { if } \alpha \in R \\ \mathbf{K} & \text { otherwise }\end{cases}
$$

The set $R$ is called the set of retractable sentences (associated to the shielded contraction $-\bullet$ ).

According to the above definition, the operator $-\rightarrow$ has the same behaviour of the contraction operator - by which is induced when it is applied to a sentence that is included in $R$, but is such that it leaves the set to be contracted unchanged whenever the belief, that is to be contracted, is not included in $R$. For this reason, the sentences included in $R$ are called retractable (sentences) and the remaining ones are designated by irretractable.

In that paper, the following rationality criteria for $R$ were proposed:

Conjunctive Completeness: If $\alpha \wedge \beta \in R$, then $\alpha \in R$ or $\beta \in R$.

Non-retractability Propagation: If $\alpha \notin R$, then $\operatorname{Cn}(\alpha) \cap R=\emptyset$.

Non-retractability Preservation: $\mathcal{L} \backslash R \subseteq \mathbf{K} \rightarrow \alpha$.

Conjunctive Completeness states that if $\alpha$ and $\beta$ are irretractable, then $\alpha \wedge \beta$ is also irretractable. In fact, in order to remove a conjunction we must remove at least one of its conjuncts. Therefore, a conjunction of two irretractable sentences must itself be irretractable. Non-retractability Propagation says that if a sentence $\alpha$ is irretractable, then all its logical consequences are also irretractable. Non-retractability Preservation states that irretractable sentences cannot be removed, independently of the (shielded) contraction performed, i.e. irretractable sentences should be (kept) in the outcome of the (shielded) contraction by any sentence.

We note that non-retractability preservation is a condition interrelating $R$ and - , rather than a property of $R$. Furthermore, if it is considered as a property of $R$ then more rigorously it should be named non-retractability preservation with respect to $\mathbf{K}$ and $\rightarrow$ since it relates $R$ and the outcomes of the contraction of $\mathbf{K}$ by means of $-\sigma$. However, we will use the shorter designation for it, since everywhere this property shall be mentioned throughout this paper it will be clear from the context which belief set and shielded contraction are being considered.

Two classes of shielded contractions were axiomatically characterized in [6]: (i) the class of shielded contractions induced by a basic AGM contraction and a set $R$ that satisfies non-retractability propagation and conjunctive completeness (Observation 2.3), and (ii) the class of shielded contractions induced by an AGM contraction and a set $R$ that satisfies non-retractability propagation, conjunctive completeness and non-retractability preservation (Observation 2.4).

Before presenting those two representation theorems we introduce the following postulates that are needed in those axiomatizations:

Relative success [27] $\mathbf{K}-\alpha=\mathbf{K}$ or $\alpha \notin \mathbf{K} \rightarrow \alpha$.

Persistence [6] If $\mathbf{K} \rightarrow \beta \vdash \beta$, then $\mathbf{K} \rightarrow \alpha \vdash \beta$.

Success propagation [6] If $\mathbf{K}-\beta \vdash \beta$ and $\vdash \beta \rightarrow \alpha$, then $\mathbf{K} \rightarrow \alpha \vdash \alpha$.

Conjunctive constancy [6] If $\mathbf{K}-\beta=\mathbf{K} \multimap \beta=\mathbf{K}$, then $\mathbf{K} \rightarrow(\alpha \wedge \beta)=\mathbf{K}$.

Relative success states that when contracting by any given sentence either that sentence is effectively removed, or the belief set is left unchanged. Persistence intuitively states that if a belief is kept when trying to contract a belief set $\mathbf{K}$ by it, then it should also be kept when contracting $\mathbf{K}$ by any other belief. Success propagation states that if a certain sentence is not removed when trying to contract a belief set by it, then the same thing happens regarding every logical consequence of that sentence. Conjunctive constancy states that if the contraction by a given conjunction causes a change, then the same thing happens when contracting by at least one of its conjuncts.

Next, and to finish this subsection, we present the two above mentioned representation theorems. 
Observation 2.3 ([6]). Let $\mathbf{K}$ be a consistent belief set and $\rightarrow$ an operator on $\mathbf{K}$. Then the following conditions are equivalent:

1. -0 satisfies closure, inclusion, vacuity, extensionality, recovery, relative success, success propagation and conjunctive constancy.

2. - is an operator of shielded contraction induced by a basic AGM contraction for $\mathbf{K}$ and a set $R \subseteq \mathcal{L}$ that satisfies non-retractability propagation and conjunctive completeness.

3. $-\rightarrow$ is an operator of shielded contraction induced by a basic AGM contraction operator for $\mathbf{K}$ and a set $R \subseteq \mathcal{L}$ that satisfies $\mathcal{L} \backslash \mathbf{K} \subseteq R$, non-retractability propagation and conjunctive completeness.

Observation 2.4 ([6]). Let $\mathbf{K}$ be a consistent belief set and $\rightarrow$ an operator on $\mathbf{K}$. Then the following conditions are equivalent:

1. $\rightarrow$ satisfies closure, inclusion, vacuity, extensionality, recovery, relative success, persistence, conjunctive inclusion and conjunctive overlap.

2. - is an operator of shielded contraction induced by an AGM contraction operator for $\mathbf{K}$ and a set $R \subseteq \mathcal{L}$ that satisfies nonretractability propagation, conjunctive completeness and non-retractability preservation.

3. - is an operator of shielded contraction induced by an AGM contraction operator for $\mathbf{K}$ and a set $R \subseteq \mathcal{L}$ that satisfies $\mathcal{L} \backslash \mathbf{K} \subseteq R$, non-retractability propagation, conjunctive completeness and non-retractability preservation.

\subsection{Belief base contraction}

The AGM model only accounts for the case when the belief state of an agent is represented by a set of sentences that is closed under logical consequence (i.e. a belief set or theory). However, not long after the publication of the AGM paper, several criticisms were made pointing out the (impractical) use of belief sets to represent belief states. Among these criticisms we can mention: (i) Belief sets are often too big (if not even infinite), this makes belief sets inadequate for computational implementations of belief change models. (ii) Belief sets make no distinction between different inconsistent belief states. (iii) Belief sets make no distinction between basic beliefs and those that are inferred by them. Several of the existing models of contraction for beliefs sets have been adapted to the case when belief states are represented by belief bases: the partial meet contractions for belief bases were presented in [19,20,17]; the kernel contractions-which can be seen as a generalization of safe contractions-were introduced in [21]; and, in [29], Mary-Anne Williams introduced the ensconcement-based contractions (of belief bases), which can be seen as adaptations to the case of belief bases of the epistemic entrenchment-based contractions.

In this subsection we recall some of the postulates and several well-known constructive models of contraction functions on belief bases.

\subsubsection{Postulates for base contraction}

We start by recalling the definition of a contraction operator in terms of postulates presented in [24].

Definition 2.5 ([24]). An operator $\div$ for a set $A$ is an operator of contraction if and only if $\div$ satisfies the following postulates:

Success: If $\nvdash \alpha$ then $A \div \alpha \nvdash \alpha$.

Inclusion: $A \div \alpha \subseteq A$.

The following postulates are well known postulates for belief base contraction ${ }^{3}$ :

Failure: If $\vdash \alpha$ then $A \div \alpha=A$.

Vacuity: If $A \nvdash \alpha$, then $A \subseteq A \div \alpha$.

Relative closure: $A \cap C n(A \div \alpha) \subseteq A \div \alpha$.

Relevance: If $\beta \in A$ and $\beta \notin A \div \alpha$ then there is some set $A^{\prime}$ such that $A \div \alpha \subseteq A^{\prime} \subseteq A$ and $\alpha \notin C n\left(A^{\prime}\right)$ but $\alpha \in C n\left(A^{\prime} \cup\{\beta\}\right)$. Core-retainment: If $\beta \in A$ and $\beta \notin A \div \alpha$ then there is some set $A^{\prime}$ such that $A^{\prime} \subseteq A$ and $\alpha \notin C n\left(A^{\prime}\right)$ but $\alpha \in \operatorname{Cn}\left(A^{\prime} \cup\{\beta\}\right)$.

Disjunctive elimination: If $\beta \in A$ and $\beta \notin A \div \alpha$, then $A \div \alpha \nvdash \alpha \vee \beta$.

Extensionality: If $\vdash \alpha \leftrightarrow \beta$, then $A \div \alpha=A \div \beta$.

Uniformity: If it holds for all subsets $A^{\prime}$ of $A$ that $\alpha \in C n\left(A^{\prime}\right)$ if and only if $\beta \in C n\left(A^{\prime}\right)$, then $A \div \alpha=A \div \beta$.

Failure [11], vacuity and relative closure [18] formalize the minimal change criteria, according to which, unnecessary loss of information should be avoided. Failure states that when contracting by a tautology, the set to be contracted is left unchanged. Vacuity ensures that nothing is lost when contracting a set by a sentence that is not a consequence of that set. Relative closure ensures that the original beliefs that are implied by the contracted set are kept. Relevance [16,19], core-retainment

\footnotetext{
${ }^{3}$ For an overview of these postulates see $[24,7]$
} 


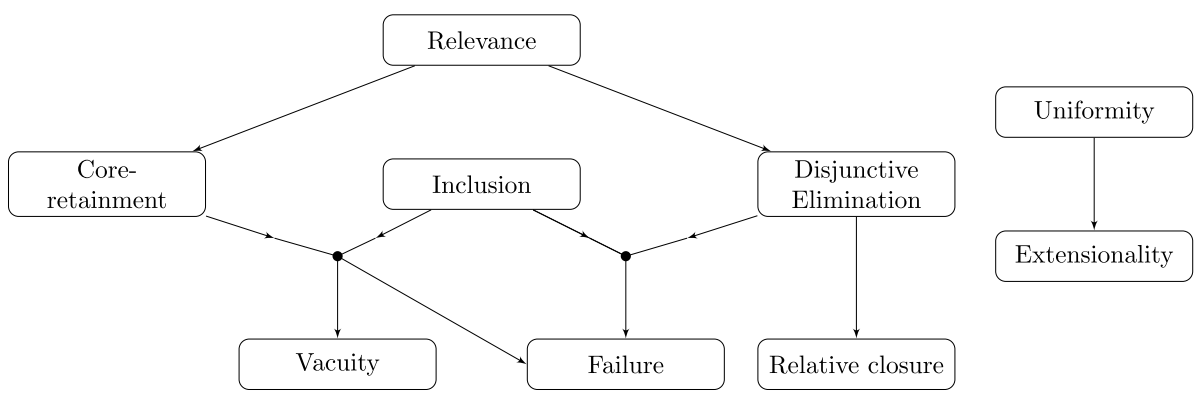

Fig. 1. Diagram of the interrelations among postulates listed in Observation 2.6.

[18] and disjunctive elimination [9] were also proposed to capture the principle of minimal change. It is interesting to notice that both core-retainment and disjunctive elimination follow from relevance. Extensionality is a formalization of the irrelevance of syntax criteria, according to which the outcome of a change should not depend on the syntax/representation used. It states that contracting by logically equivalent sentences produces the same outcome. Uniformity [19] is a stronger version of extensionality, it states that when $\alpha$ and $\beta$ are two sentences implied by exactly the same subsets of $A$, then the result of contracting $A$ by $\alpha$ is identical to the outcome of contracting $A$ by $\beta$.

In the following observation we present some relations between the postulates presented above.

Observation 2.6. Let $A$ be a belief base and $\div$ an operator on $A$. Then:

(a) [24] If $\div$ satisfies relevance, then it satisfies relative closure and core-retainment.

(b) [24] If $\div$ satisfies inclusion and core-retainment, then it satisfies failure and vacuity.

(c) [24] If $\div$ satisfies uniformity, then it satisfies extensionality.

(d) [9] If $\div$ satisfies disjunctive elimination, then it satisfies relative closure. If $\div$ also satisfies inclusion, then it satisfies failure.

(e) [9] If $\div$ satisfies relevance, then it satisfies disjunctive elimination.

In Fig. 1 we present a diagram that summarizes all the interrelations among postulates that were stated in the above observation.

\subsubsection{Partial meet contraction}

The outcome of contracting a belief base $A$ by a sentence $\alpha$ should be a subset of $A$ that fails to imply $\alpha$ (whenever that is possible). In the outcome of this contraction no elements of $A$ should be unnecessarily removed. Hence a base contraction function can be constructed using remainder sets, i.e., maximal subsets of $A$ that fail to imply $\alpha$ :

Definition 2.7 ([2]). Let $A$ be a belief base and $\alpha$ a sentence. The set $A \perp \alpha$ ( $A$ remainder $\alpha$ ) is the set of sets such that $B \in A \perp \alpha$ if and only if:

1. $B \subseteq A$.

2. $B \nvdash \alpha$.

3. There is no set $B^{\prime}$ such that $B \subset B^{\prime} \subseteq A$ and $B^{\prime} \nvdash \alpha$.

Definition 2.8 ([1]). Let $A$ be a belief base. A selection function for $A$ is a function $\gamma$ such that for all sentences $\alpha$ :

1. If $A \perp \alpha$ is non-empty, then $\gamma(A \perp \alpha)$ is a non-empty subset of $A \perp \alpha$.

2. If $A \perp \alpha$ is empty, then $\gamma(A \perp \alpha)=\{A\}$.

Definition 2.9 ([1,17]). The partial meet contraction operator on $A$ based on a selection function $\gamma$ is the operator $-\gamma$ such that for all sentences $\alpha$ :

$$
A-\gamma \alpha=\cap \gamma(A \perp \alpha)
$$

An operator - for a set $A$ is a partial meet contraction if and only if there is a selection function $\gamma$ for $A$ such that $A-\alpha=A-\gamma \alpha$ for all sentences $\alpha$.

Hansson characterized partial meet contractions defined on belief bases in terms of postulates:

Observation 2.10 ([17]). Let $A$ be a belief base. An operator $\div$ on $A$ is a partial meet contraction function for $A$ if and only if it satisfies success, inclusion, uniformity and relevance. 


\subsubsection{Kernel contraction}

In [21] Hansson introduced Kernel Contraction, a generalization of Safe Contraction [3]. It is based on a selection among the sentences of a set $A$ that contribute effectively to imply $\alpha$; and on how to use this selection in contracting by $\alpha$. Formally:

Definition 2.11 ([21]). Let $A$ be a set in $\mathcal{L}$ and $\alpha$ a sentence. Then $A \Perp \alpha$ is the set such that $B \in A \Perp \alpha$ if and only if:

1. $B \subseteq A$.

2. $B \vdash \alpha$.

3. If $B^{\prime} \subset B$ then $B^{\prime} \nvdash \alpha$.

$A \Perp \alpha$ is called the kernel set of $A$ with respect to $\alpha$ and its elements are the $\alpha$-kernels of $A$.

To contract a belief $\alpha$ from a set $A$ one must give up sentences in each $\alpha$-kernel, otherwise $\alpha$ would continue being implied by $A$. The so-called incision functions select the beliefs to be discarded.

Definition 2.12 ([21]). Let $A$ be a set of sentences. Let $A \Perp \alpha$ be the kernel set of $A$ with respect to $\alpha$. An incision function $\sigma$ for $A$ is a function such that for all sentences $\alpha$ :

1. $\sigma(A \Perp \alpha) \subseteq \bigcup(A \Perp \alpha)$.

2. If $\emptyset \neq B \in A \Perp \alpha$, then $B \cap \sigma(A \Perp \alpha) \neq \emptyset$.

Definition 2.13 ([21]). Let $A$ be a set of sentences and $\sigma$ an incision function for $A$. The kernel contraction $-\sigma$ for $A$ is defined as:

$$
A-\sigma \alpha=A \backslash \sigma(A \Perp \alpha) \text {. }
$$

An operator - for a set $A$ is a kernel contraction if and only if there is an incision function $\sigma$ for $A$ such that $A-\alpha=$ $A-{ }_{\sigma} \alpha$ for all sentences $\alpha$.

Hansson also provided an axiomatic characterization for kernel contractions defined on belief bases.

Observation 2.14 ([21]). Let $A$ be a belief base. An operator $\div$ on $A$ is a kernel contraction if and only if it satisfies success, inclusion, uniformity and core-retainment.

In the following we recall a more conservative type of kernel contractions, namely smooth kernel contractions and their axiomatic characterization. Sometimes, when contracting a set by a kernel contraction, some beliefs are removed without reason. For example if $\beta \in A$ and $\beta \in C n(A \div \alpha)$, then $\beta$ should also be in $A \div \alpha$. This can be solved if we ensure that an operator of kernel contraction satisfies relative closure. This can be done if we impose the condition expressed in the following definition to incision functions.

Definition 2.15 ([21]). An incision function $\sigma$ for a set $A$ is smooth if and only if it holds for all subsets $A^{\prime}$ of $A$ that if $A^{\prime} \vdash \beta$ and $\beta \in \sigma(A \Perp \alpha)$ then $A^{\prime} \cap \sigma(A \Perp \alpha) \neq \emptyset$.

A kernel contraction is smooth if and only if it is based on a smooth incision function.

The following observation presents an axiomatic characterization for the class of smooth kernel contractions.

Observation 2.16 ([21]). Let $A$ be a belief base. An operator $\div$ on $A$ is a smooth kernel contraction if and only if it satisfies success, inclusion, uniformity, core-retainment and relative closure.

\subsubsection{Basic AGM-generated base contraction}

In what follows we recall the definition of another kind of base contractions as well as their axiomatic characterization.

Definition 2.17 ([9]). Let $A$ be a belief base. An operator $\div$ for $A$ is a basic AGM-generated base contraction if and only if, for all $\alpha \in \mathcal{L}$ :

$$
A \div \alpha=(C n(A)-\alpha) \cap A
$$

where - is a basic AGM contraction (i.e. an operator that satisfies the basic AGM postulates for contraction) on $C n(A){ }^{4}$

\footnotetext{
${ }^{4}$ In [9] these operators were designated by basic related-AGM base contractions.
} 
Observation 2.18 ([9]). Let $A$ be a belief base. An operator $\div$ on $A$ is a basic AGM-generated base contraction if and only if it satisfies success, inclusion, vacuity, extensionality and disjunctive elimination.

The following observation exposes interrelations among the different classes of contractions recalled in this subsection which follow trivially from their axiomatic characterizations presented in Observations 2.10, $2.14,2.16$ and 2.18 and the interrelations among postulates that we presented in Observations 2.6 (and that are represented in the diagram of Fig. 1).

Observation 2.19. Let $A$ be a belief base and $\div$ be a contraction operator on $A$. Then:

(a) If $\div$ is an operator of partial meet contraction, then it is an operator of smooth kernel contraction.

(b) If $\div$ is an operator of smooth kernel contraction, then it is an operator of kernel contraction.

(c) If $\div$ is an operator of partial meet contraction, then it is an operator of basic AGM-generated base contraction.

\subsection{Shielded contraction for belief bases}

In this subsection we summarize the main concepts and results, so far presented in the literature, concerning the adaptation to the case of belief bases of the operators of shielded contraction (on belief sets) that we recalled in Subsection 2.2 .

In [10], Fermé, Mikalef and Taboada adapted the definition of shielded contraction that we recalled in Definition 2.2 to the case of belief bases.

Definition 2.20 ([10]). Let $A$ be a belief base. An operator $\sim$ on $A$ is a shielded partial meet base contraction if there exist a partial meet contraction $\div$ on $A$ and a set of (retractable) sentences $R$ such that, for all $\alpha \in \mathcal{L}$ :

$$
A \sim \alpha= \begin{cases}A \div \alpha & \text { if } \alpha \in R \\ A & \text { otherwise }\end{cases}
$$

The set $R$ is called the set of retractable sentences or the retractable set associated to $\sim$.

In [10] the operators presented in the above definition were designated simply by shielded base contraction but here it is convenient to use this alternative designation, because in this paper we shall use the expression shielded base contraction to designate a wider class of functions (cf. Definition 3.1).

\subsubsection{Postulates for shielded contraction on belief bases}

In [10] the postulates (that we recalled in Subsection 2.2) proposed in [6] for shielded contractions on belief sets were adapted to the belief bases context:

Relative success: $A \sim \alpha=A$ or $\alpha \notin C n(A \sim \alpha)$.

Persistence: If $A \sim \beta \vdash \beta$, then $A \sim \alpha \vdash \beta$.

Success propagation: If $A \sim \beta \vdash \beta$ and $\vdash \beta \rightarrow \alpha$, then $A \sim \alpha \vdash \alpha$.

Conjunctive constancy: If $A \sim \alpha=A \sim \beta=A$, then $A \sim(\alpha \wedge \beta)=A$.

The following observations illustrate some relations among the postulates presented above.

Observation 2.21 ([6]). Let $\sim$ be an operator on A. If satisfies persistence, then it satisfies success propagation.

Observation 2.22. Let $\sim$ be an operator on $A$.

(a) If $\sim$ satisfies relative success, then it satisfies failure.

(b) If satisfies inclusion, vacuity, persistence and relative success, then it satisfies conjunctive constancy.

\subsubsection{Properties of the set of retractable sentences}

In [10] the properties that were considered as properties that may be desirable from a set $R$ of retractable sentences were the following:

Non-retractability Propagation: If $\alpha \notin R$, then $C n(\alpha) \cap R=\emptyset$.

Conjunctive Completeness: If $\alpha \wedge \beta \in R$, then $\alpha \in R$ or $\beta \in R$. 
Non-retractability Preservation: $\mathcal{L} \backslash R \subseteq C n(A \sim \alpha)$.

Non-retractability of Tautology: $R \cap C n(\emptyset)=\emptyset$.

The first three ones of the above properties were proposed in [6]. The first two ones of the above properties were recalled in Subsection 2.2. Non-retractability preservation is a direct adaptation to the case of belief bases of the property with the same designation that was proposed in [6] for belief sets (and was recalled in Subsection 2.2). Non-retractability of tautology states that tautologies should be irretractable sentences.

\subsubsection{An axiomatic characterization of shielded partial meet base contractions}

In [10], the following axiomatic characterization was obtained for the class of shielded partial meet base contractions whose associated retractable set satisfies non-retractability propagation and non-retractability preservation.

Observation 2.23 ([10]). Let $A$ be a belief base and $\sim$ an operator on $A$. Then the following conditions are equivalent:

(a) satisfies relative success, persistence, inclusion, relevance and uniformity.

(b) $\sim$ is an operator of shielded partial meet base contraction whose associated retractable set $R \subseteq \mathcal{L}$ satisfies non-retractability propagation and non-retractability preservation.

Actually, we must note that in the representation theorem presented in [10] the list of postulates consists of the postulates mentioned in (a) together with vacuity and conjunctive constancy. However, according to Observations 2.6 and 2.22, the latter two mentioned postulates follow from the remaining ones. Thus the axiomatic characterization presented in the above observation can, in fact, be seen as a refinement of the one presented in [10].

\section{Shielded base contraction}

In this paper we extend the definition of shielded base contraction proposed in [10], by defining operators of shielded base contraction in terms of other kinds of contraction functions (rather than only by means of partial meet contractions). In this section we start by introducing a definition for shielded base contractions which constitutes a generalization of the class of operators presented in Definition 2.20. After that we propose some new properties that are adequate to require from a set of retractable sentences and we study the interrelations among these properties and the properties associated to sets of retractable sentences in [6] and in [10] (which we have recalled in the previous section).

\subsection{A general definition for shielded base contractions}

The following definition presents a class of shielded base contractions which generalizes the class mentioned in Definition 2.20 in the sense that the underlying contraction does not need to be a partial meet contraction.

Definition 3.1. Let $\div$ be a contraction operator on a belief base $A$ (i.e. an operator that satisfies success and inclusion). Let $R$ be a set of sentences (the associated set of retractable sentences). Then $\sim$ is the shielded base contraction induced by $\div$ and $R$ if and only if:

$$
A \sim \alpha= \begin{cases}A \div \alpha & \text { if } \alpha \in R \\ A & \text { otherwise }\end{cases}
$$

We note that if $\sim$ is a shielded contraction induced by a contraction operator and the set $R=\mathcal{L} \backslash C n(\emptyset)$, then $\sim$ is a (standard) contraction operator that satisfies failure.

\subsection{The set of retractable sentences}

In this subsection we propose some new properties that may naturally be required from a set of retractable sentences and, after that, we present some results exposing interrelations among different properties associated to sets of retractable sentences.

\subsubsection{New properties for the set of retractable sentences}

We start by introducing the following property which states that two logically equivalent sentences should be both retractable or both irretractable.

Retractability of Logical Equivalents: If $\vdash \alpha \leftrightarrow \beta$, then $\alpha \in R$ if and only if $\beta \in R$.

Another property that is natural to expect is the following one, which attests that a conjunction is retractable as long as one of its conjuncts is so. 
Converse Conjunctive Completeness: If $\alpha \in R$, then $\alpha \wedge \beta \in R$.

Additionally, we propose some other properties interconnecting $R$ and $A$ that are natural to expect:

Uniform Retractability: If it holds for all subsets $A^{\prime}$ of $A$ that $\alpha \in C n\left(A^{\prime}\right)$ if and only if $\beta \in C n\left(A^{\prime}\right)$, then $\alpha \in R$ if and only if $\beta \in R$.

Uniform retractability states that if two sentences $\alpha$ and $\beta$ are implied by exactly the same subsets of $A$, then they are both retractable or both irretractable.

Non-retractability Upper Bounding: $\mathcal{L} \backslash R \subseteq C n(A)$.

Non-retractability upper bounding states that all irretractable sentences are deducible from the set to be contracted. We notice that this property is equivalent to $\mathcal{L} \backslash C n(A) \subseteq R$ which is mentioned in the representation results of [6] that we have recalled in Subsection 2.2.5

At this point we propose the following condition that relates a set of retractable sentences $R$ and a contraction function $\div$ :

If $\alpha \notin R$ and $\beta \in R$, then $A \div \beta \vdash \alpha$.

The intuition underlying this condition is the following:

Ideally the irretractable sentences should not be removed from the belief base when a shielded contraction (by any sentence) is performed. Then, if a set (of retractable sentences) $R$ and a contraction $\div$ are intended to be used to define a shielded contraction it should hold that if a sentence is not in $R$ (i.e., is considered irretractable), then it should not be removed when using $\div$ to contract by a sentence included in $R$ (i.e., by a sentence that is considered retractable). ${ }^{6}$ The following example clarifies this reasoning.

Example 3.2. Let $A=\{p, q\}$ and $R=\mathcal{L} \backslash C n(p)$, where $C n$ is purely truth-functional. ${ }^{7}$ Consider a contraction operator $\div$ such that $A \div(p \wedge q)=\{q\}$. If $\sim$ is the shielded contraction induced by $\div$ and $R$, it holds that:

$$
A \sim(p \wedge q)=A \div(p \wedge q)=\{q\} \nvdash p .
$$

This contradicts one of the main goals underlying the concept of "irretractability": irretractable sentences should be kept (more precisely, should be implied) after performing the related shielded contraction (by any sentence). We note that this happens because $R$ and $\div$ do not satisfy condition $(\mathbf{R}-\div)$. Indeed, $p \notin R$ and $p \wedge q \in R$ but $A \div(p \wedge q) \nvdash p$.

From a different perspective, we can say that condition $(\mathbf{R}-\div)$ imposes that $R$ and $\div$ are such that the complement of $R$ consists precisely of the sentences which are more difficult to remove by means of $\div$.

We note that if $R$ and $\div$ satisfy condition $(\mathbf{R}-\div)$, then the shielded contraction that is induced by $R$ and $\div$ satisfies the persistence postulate. This will be stated more formally in Theorem 3.8 (b).

Throughout this paper we shall often say, by abuse of language, that $R$ satisfies condition $(\mathbf{R}-\div)$, instead of saying that $R$ and $\div$ satisfy condition $(\mathbf{R}-\div)$.

\subsubsection{Interrelations among properties for the set of retractable sentences}

The following observation highlights that if a set $R$ of retractable sentences satisfies non-retractability of tautology, non-retractability propagation and conjunctive completeness, then its complement $\mathcal{L} \backslash R$, the set of irretractable sentences, is logically closed.

Observation 3.3. Let $R$ be a set that satisfies non-retractability of tautology, non-retractability propagation and conjunctive completeness. Then:

$$
\alpha \in \mathcal{L} \backslash R \text { if and only if } \mathcal{L} \backslash R \vdash \alpha .
$$

\footnotetext{
5 We note that more rigorously the expression "with respect to $A$ " should be added to the designation of the last two properties presented (namely, uniform retractability and non-retractability upper bounding), since these relate $R$ and $A$. However we will use the shorter designation of these properties since there is no risk of ambiguity whenever these properties are mentioned along this paper.

6 Note that if $\beta \notin R$ and $\sim$ is a shielded base contraction operator on a set $A$ induced by a contraction operator and $R$, then $A \sim \beta=A$. Therefore if $R$ satisfies non-retractability upper bounding then $A \sim \beta \vdash \alpha$ for every irretractable sentence $\alpha$.

7 Note that the set $R$ satisfies non-retractability of tautology, non-retractability propagation, conjunctive completeness, uniform retractability and nonretractability upper bounding.
} 
We note that, if $R \neq \mathcal{L}$ satisfies non-retractability propagation, then it is not logically closed i.e., the fact that $R \vdash \alpha$ does not imply that $\alpha \in R$. To see this it is enough to note that if $p \in R$ and $q \notin R$, then $R \vdash p \vee q$. But, by non-retractability propagation, $p \vee q \notin R$.

The following observation illustrates that retractability of logical equivalents follows from uniform retractability and also follows from non-retractability propagation. It also highlights that converse conjunctive completeness is implied by nonretractability propagation.

Observation 3.4. Let $R$ be a set of sentences. Then:

(a) If $R$ satisfies uniform retractability (with respect to some set of sentences $A$ ), then $R$ satisfies retractability of logical equivalents.

(b) If $R$ satisfies non-retractability propagation, then $R$ satisfies retractability of logical equivalents and converse conjunctive completeness.

The following theorem presents an explicit definition for the set of retractable sentences $R$ in terms of the shielded contraction operator $\sim$, which is induced by it, provided that $R$ satisfies non-retractability of tautology and non-retractability upper bounding.

Theorem 3.5. Let $A$ be a belief base and $\sim$ be a shielded base contraction induced by a contraction operator on $A$ and $a$ set $R \subseteq \mathcal{L}$. Then $R$ satisfies non-retractability of tautology and non-retractability upper bounding if and only if $R=\{\alpha: A \sim \alpha \nvdash \alpha\}$.

The following observation exposes that a shielded contraction $\sim$ is induced by a contraction operator $\div$ and a set $R$ that satisfies non-retractability upper bounding and which are such that condition ( $\mathbf{R}-\div)$ holds, if and only if $R$ satisfies non-retractability preservation.

Observation 3.6. Let $A$ be a belief base and $\sim$ be a shielded base contraction induced by a contraction operator $\div$ on $A$ and a set $R \subseteq \mathcal{L}$. Then, $R$ satisfies non-retractability preservation if and only if $R$ satisfies non-retractability upper bounding and $\div$ and $R$ satisfy condition $(\mathbf{R}-\div)$.

According to the above result, given a shielded base contraction $\sim$ induced by a contraction operator $\div$ and a set (of retractable) sentences $R$, in the presence of non-retractability upper bounding, the properties of non-retractability preservation and $(\mathbf{R}-\div)$ are equivalent. Nevertheless, we note that, condition $(\mathbf{R}-\div)$ relates two structures (namely $\div$ and $R$ ) that are independent of each other, while, on the other hand, non-retractability preservation presents a relation between a shielded contraction $\sim$ and its associated set of retractable sentences. Therefore, in the process of constructing a shielded base contraction (as exposed in Definition 3.1) it is more natural to consider condition ( $\mathbf{R}-\div$ ) than non-retractability preservation.

The following observation exposes some other relations among the properties of a set of retractable sentences.

Observation 3.7. Let $A$ be $a$ belief base and $\sim$ be a shielded base contraction induced by a contraction operator on $A$ and a set $R \subseteq \mathcal{L}$. If $R$ satisfies non-retractability preservation and non-retractability of tautology, then $R$ satisfies conjunctive completeness, non-retractability propagation, uniform retractability and retractability of logical equivalents.

\subsection{Relations between base contractions and shielded base contractions}

The following theorem illustrates some properties that an operator of shielded base contraction, induced by a contraction operator $\div$ and a set $R$, satisfies whenever $\div$ and $R$ satisfy some given properties.

Theorem 3.8. Let $A$ be a belief base, $\div$ be a contraction on $A, R \subseteq \mathcal{L}$, and $\sim$ be the shielded base contraction induced by $\div$ and $R$. Then $^{8}$ :

\footnotetext{
8 The schema presented in this theorem (and whenever a similar schema is used) should be read as follows: Let $A$ be a belief base, $\div$ be a contraction on $A, R \subseteq \mathcal{L}$, and $\sim$ be the shielded base contraction induced by $\div$ and $R$. Then:
}

(a) It holds that:
1. $\sim$ satisfies inclusion.
2 . If $\div$ satisfies vacuity, then $\sim$ satisfies vacuity.
3 . If $\div$ satisfies failure, then $\sim$ satisfies relative success. 
(a) It holds that:

\begin{tabular}{cc}
\hline If $\div$ satisfies & then $\sim$ satisfies \\
\hline- & inclusion \\
\hline vacuity & vacuity \\
\hline failure & relative success \\
\hline relative closure & relative closure \\
\hline relevance & relevance \\
\hline core-retainment & core-retainment \\
\hline disjunctive elimination & disjunctive elimination \\
\hline
\end{tabular}

(b) If $R$ and $\div$ satisfy condition $(\mathbf{R}-\div)$, then:

\begin{tabular}{cc}
\hline If $\div$ satisfies & then $\sim$ satisfies \\
\hline- & persistence \\
\hline failure and extensionality & extensionality \\
\hline failure and uniformity & uniformity \\
\hline
\end{tabular}

(c) If $R$ satisfies non-retractability preservation, then:

\begin{tabular}{cc}
\hline If $\div$ satisfies & then $\sim$ satisfies \\
\hline- & persistence \\
\hline failure and extensionality & extensionality \\
\hline failure and uniformity & uniformity \\
\hline
\end{tabular}

(d) If $R$ satisfies non-retractability of tautology, then:

\begin{tabular}{cc}
\hline If $\div$ satisfies & then $\sim$ satisfies \\
\hline- & relative success
\end{tabular}

(e) If $R$ satisfies retractability of logical equivalents, then:

\begin{tabular}{lc}
\hline If $\div$ satisfies & then $\sim$ satisfies \\
\hline extensionality & extensionality \\
\hline
\end{tabular}

(f) If $R$ satisfies uniform retractability, then:

\begin{tabular}{lc}
\hline If $\div$ satisfies & then $\sim$ satisfies \\
\hline uniformity & uniformity \\
\hline
\end{tabular}

(g) If $R$ satisfies non-retractability propagation, then:

\begin{tabular}{lc}
\hline If $\div$ satisfies & then $\sim$ satisfies \\
\hline- & success propagation \\
\hline extensionality & extensionality \\
\hline
\end{tabular}

(h) If $R$ satisfies conjunctive completeness and retractability of logical equivalents (or uniform retractability), then:

\begin{tabular}{lc}
\hline If $\div$ satisfies & then $\sim$ satisfies \\
\hline vacuity and failure & conjunctive constancy \\
\hline
\end{tabular}

(i) If $R$ satisfies non-retractability propagation and conjunctive completeness, then:

\begin{tabular}{lc}
\hline If $\div$ satisfies & then $\sim$ satisfies \\
\hline vacuity and extensionality (or uniformity) & conjunctive constancy \\
\hline
\end{tabular}


At this point we note that, given a shielded contraction $\sim$, we can use it to define a set $R$ of sentences that may be considered retractable in that context. As Theorem 3.5 suggests, a natural way to define such a set is by $R=\{\alpha: A \sim \alpha \nvdash \alpha\}$. The next theorem illustrates some properties that this set $R$ satisfies whenever $\sim$ satisfies some of the postulates presented in Subsections 2.3 and 2.4.

Theorem 3.9. Let $A$ be a belief base, $\sim$ be an operator on $A$ and $R=\{\alpha: A \sim \alpha \nvdash \alpha\}$. Then:

(a) It holds that:

\begin{tabular}{cc}
\hline If $\sim$ satisfies & then R satisfies \\
\hline- & non-retractability of tautology \\
\hline inclusion & non-retractability upper bounding \\
\hline extensionality & retractability of logical equivalents \\
\hline $\begin{array}{c}\text { inclusion and uniformity } \\
\text { success propagation }\end{array}$ & uniform retractability \\
\hline $\begin{array}{c}\text { relative success and } \\
\text { conjunctive constancy }\end{array}$ & non-retractability propagation \\
\hline persistence & $\begin{array}{c}\text { non-retractability preservation, conjunctive } \\
\text { completeness, non-retractability propagation } \\
\text { and retractability of logical equivalents }\end{array}$ \\
\hline persistence and inclusion & uniform retractability \\
\hline
\end{tabular}

(b) If $\sim$ is a shielded base contraction induced by a contraction operator $\div$ and $R$, then:

\begin{tabular}{lc}
\hline If $\sim$ satisfies & then $R$ satisfies \\
\hline persistence & non-retractability upper \\
& bounding and condition $(\mathbf{R}-\div)$ \\
\hline
\end{tabular}

We have already explored the properties that an operator of shielded base contraction induced by an operator $\div$ and a set $R$ satisfies whenever $\div$ and $R$ satisfy some given properties. In the next theorem we will see that it is possible to construct an operator $\div$ in terms of $\sim$ and $R=\{\alpha: A \sim \alpha \nvdash \alpha\}$ and investigate the properties that such an operator satisfies taking into account the properties satisfied by $\sim$.

Theorem 3.10. Let $A$ be a belief base, $\sim$ be an operator on $A, R=\{\alpha: A \sim \alpha \nvdash \alpha\}$ and $\div$ be the operator defined (for all $\alpha$ ) by the following condition:

$$
A \div \alpha= \begin{cases}A \sim \alpha & \text { if } \alpha \in R \\ A-\alpha & \text { otherwise }\end{cases}
$$

where - is an operator on A that satisfies success, inclusion, uniformity and relevance. ${ }^{9}$ It holds that:

(a) If $\sim$ satisfies relative success and inclusion, then $\div$ is a contraction operator and $\sim$ is the shielded base contraction induced by $\div$ and $R$.

(b) It holds that:

\begin{tabular}{cc}
\hline If $\sim$ satisfies & then $\div$ satisfies \\
\hline vacuity & vacuity \\
\hline extensionality & extensionality \\
\hline failure & failure \\
\hline relative closure & relative closure \\
\hline inclusion and uniformity & uniformity \\
\hline relevance & relevance \\
\hline core-retainment & core-retainment \\
\hline disjunctive elimination & disjunctive elimination \\
\hline
\end{tabular}

$\overline{9}$ Note that - can be, for example, the operator defined (for all $\alpha$ ) by $A-\alpha=A \backslash \bigcup(A \Perp \alpha)$ or any other partial meet contraction (cf. Observation 2.10). 
(c) If $\sim$ satisfies persistence, then $\div$ and $R$ satisfy condition $(\mathbf{R}-\div)$.

\section{Axiomatic characterizations of different kinds of shielded base contractions}

In this section we propose and axiomatically characterize several classes of shielded contraction functions. We start by presenting, in Subsection 4.1 a representation theorem for the operators that satisfy relative success and inclusion, which constitute the most general class of shielded contractions that we will consider. Afterwards in Subsections 4.2-4.5 we consider some more specific classes of shielded contractions. More precisely we consider the classes of shielded contractions induced by different well-known kinds of contraction functions (namely, partial meet, (smooth) kernel and basic AGM-generated base contractions) and by several alternative types of sets of retractable sentences (i.e. considering different sets of properties associated to the related set of retractable sentences). We note that the proofs provided in the Appendix for the representation theorems included here rely very strongly on the results presented in the previous section.

All the Subsections 4.2-4.5 have a similar structure. In each one of these subsections firstly a theorem is presented which provides axiomatic characterizations for five classes of shielded contractions all induced by a same kind of contraction function but each of them with a different type of associated set of retractable sentences. Afterwards, a definition is presented where a designation is proposed for each one of the operators that were axiomatically characterized in the previously presented theorem. The reason for providing a designation for each of the considered classes of operators only after presenting the corresponding axiomatic characterization is the fact that the designation proposed for each class is based on the names of some of the postulates that are included in that axiomatic characterization.

\subsection{Basic shielded base contractions}

In Definition 3.1 we have introduced, from a constructive perspective, the concept of shielded base contraction induced by a contraction operator $\div$ and a set of retractable sentences $R$. At this point we notice that if, alternatively, we wished to define shielded base contractions in terms of postulates then, having in mind (i) the definition for contraction recalled in Definition 2.5, and (ii) the postulates for shielded contraction presented in Subsections 2.3 and 2.4, the most natural proposal would be to define a shielded base contraction as an operator that satisfies the postulates of relative success and inclusion.

It follows trivially from Definition 3.1 that any shielded contraction satisfies inclusion. However, somehow surprisingly, a shielded contraction operator induced by a (general) contraction operator $\div$ (i.e. an operator $\div$ that satisfies success and inclusion) and a set of retractable sentences $R \subseteq \mathcal{L}$ (which is not required to satisfy any properties at all) does not satisfy the postulate of relative success. Indeed, in order to assure that a shielded base contraction $\sim$ induced by a contraction $\div$ and a set $R$ satisfies relative success, it is necessary to impose that $\div$ satisfies the postulate of failure and/or that the set $R$ satisfies non-retractability of tautology. This fact is formally stated in the following theorem.

Theorem 4.1. Let $A$ be a belief base and $\sim$ an operator on A. Then the following conditions are equivalent:

1. $\sim$ satisfies relative success and inclusion.

2. $\sim$ is an operator of shielded base contraction induced by a contraction operator and a set $R \subseteq \mathcal{L}$ that satisfies non-retractability of tautology.

3. $\sim$ is an operator of shielded base contraction induced by a contraction operator that satisfies failure and a set $R \subseteq \mathcal{L}$.

Having in mind the above theorem and the discussion that preceded it, we are led to consider that the most general kind of shielded contractions that are worth considering (in the sense that these are the most general operators that satisfy the minimal set of properties that are intuitively associated to the notion of shielded contraction) are the ones that we introduce in the following definition.

Definition 4.2. A shielded base contraction $\sim$ on a belief base $A$ induced by a contraction $\div$ and a set $R \subseteq \mathcal{L}$ is a basic shielded contraction if and only if $\div$ satisfies failure or $R$ satisfies non-retractability of tautology.

In the following result we present an axiomatic characterization for the basic shielded contractions, which follows trivially from Theorem 4.1 .

Corollary 4.3. Let $\sim$ be an operator on A. Then $\sim$ is a basic shielded contraction if and only if $\sim$ satisfies relative success and inclusion.

In the remainder of this section we will obtain axiomatic characterizations for other less general classes of shielded contractions (which are strict subclasses of the class of basic shielded contractions). More precisely, we will consider the shielded contractions on belief bases induced by partial meet contractions, by (smooth) kernel contractions and by basic AGM-generated base contractions and, additionally, we will take into account different sets of properties regarding the associated set of retractable sentences $R$. 


\subsection{Shielded partial meet base contractions}

The following theorem presents axiomatic characterizations for five kinds of operators of shielded contraction on belief bases. All these operators are induced by partial meet contractions but each one of them has a different type of associated set of retractable sentences. ${ }^{10}$

Theorem 4.4. Let $A$ be a belief base and $\sim$ an operator on A. Then:

\begin{tabular}{cc}
\hline $\begin{array}{c}\sim \text { is an operator of shielded base contraction induced by a } \\
\text { partial meet contraction } \div \text { and a set } R \subseteq \mathcal{L} \text { that satisfies }\end{array}$ & $\begin{array}{c}\text { if and only if } \sim \text { satisfies relative success, } \\
\text { inclusion, uniformity, relevance and }\end{array}$ \\
\hline uniform retractability & - \\
\hline uniform retractability and non-retractability propagation & success propagation \\
\hline uniform retractability and conjunctive completeness & conjunctive constancy \\
\hline $\begin{array}{c}\text { uniform retractability, non-retractability propagation and } \\
\text { conjunctive completeness }\end{array}$ & success propagation and conjunctive constancy \\
\hline condition $(\mathrm{R}-\div)$ & persistence \\
\hline
\end{tabular}

In the following definition we attribute designations to the different kinds of shielded contractions that were axiomatically characterized in the above theorem.

Definition 4.5. A shielded base contraction $\sim$ on a belief base $A$ induced by a partial meet contraction $\div$ and a set $R \subseteq \mathcal{L}$ is a:

\begin{tabular}{cc}
\hline Designation & if and only if $R$ satisfies \\
\hline Shielded partial meet contraction (SPMC) & uniform retractability \\
\hline $\begin{array}{c}\text { Success propagant shielded partial meet contraction } \\
\text { (SP-SPMC) }\end{array}$ & $\begin{array}{c}\text { uniform retractability and non-retractability } \\
\text { propagation }\end{array}$ \\
\hline $\begin{array}{c}\text { Conjunctive constant shielded partial meet contraction } \\
\text { (CC-SPMC) }\end{array}$ & $\begin{array}{c}\text { uniform retractability and conjunctive } \\
\text { completeness }\end{array}$ \\
\hline $\begin{array}{c}\text { Success propagant conjunctive constant shielded partial } \\
\text { meet contraction (SP+CC-SPMC) }\end{array}$ & $\begin{array}{c}\text { uniform retractability, non-retr. propagation } \\
\text { and conjunctive completeness }\end{array}$ \\
\hline Persistent shielded partial meet contraction \\
(P-SPMC)
\end{tabular}

Throughout this paper we will sometimes use the acronyms introduced in the first column of the table above, to designate the whole class of the corresponding kind of operators (instead of only one of the elements of that class). It will always be clear by the context whether the acronym is being used to denote a class of operators or a single operator.

It is worth to mention here that, since in the proof of the right-to-left part of Theorem 4.4 we used the set $R=\{\alpha$ : $A \sim \alpha \nvdash \alpha\}$, it follows from Observation 3.4 (a) and Theorem 3.9 that in each row of the tables presented in Theorem 4.4 and in Definition 4.5, to the list of properties of $R$ (there presented) we can add the following ones: non-retractability of tautology, non-retractability upper-bounding and retractability of logical equivalents. Furthermore, in the case of the last row, besides the three properties mentioned above, uniform retractability, non-retractability propagation, conjunctive completeness and non-retractability preservation can also be added to the list of properties of $R$ (there presented). In fact, according to Observation 3.6, in the last row of the tables presented in Theorem 4.4 and in Definition 4.5 , condition ( $\mathbf{R}-\div$ ) can even be replaced by non-retractability preservation.

We note that it follows from the above remark that there are several alternative (equivalent) definitions for the classes introduced in Definition 4.5, more precisely several equivalent definitions, but each one with a different set of properties associated to the set $R$.

This same situation occurs regarding the classes of shielded contractions introduced in Definitions $4.9,4.12$ and 4.15 (that will appear in the following subsections).

The following corollary follows trivially from the above definition and Theorem 4.4.

Corollary 4.6. Let $\sim$ be an operator on A. Then:

\footnotetext{
10 The definition and axiomatic characterization of partial meet base contractions were recalled in Subsubsection 2.3.2.
} 


\begin{tabular}{cc}
\hline$\sim$ is a & $\begin{array}{c}\text { if and only if } \sim \text { satisfies relative success, } \\
\text { inclusion, uniformity, relevance and }\end{array}$ \\
\hline$S P M C$ & - \\
\hline$S P-S P M C$ & success propagation \\
\hline$C C-S P M C$ & conjunctive constancy \\
\hline$S P+C C-S P M C$ & success propagation and conjunctive constancy \\
\hline$P-S P M C$ & persistence \\
\hline
\end{tabular}

At this point it is worth to notice that the axiomatic characterization given in the last row of the above table is identical to the axiomatic characterization presented in [10] (that we recalled in Observation 2.23). Therefore, the class formed by the operators designated in Definition 4.5 by persistent shielded partial meet contraction coincides with the class of shielded contractions that was axiomatically characterized in [10].

Next we present examples of shielded contraction operators that belong to some of the classes introduced in Definition 4.5 but do not belong to others. These examples allow us to conclude that the classes mentioned in Definition 4.5 are all different from each other.

Example 4.7. Let $A=\{p, q\}, C n$ be purely truth-functional and $\div$ be a partial meet contraction on $A$ such that $A \div(p \wedge q)=$ $\{p\}$ and $A \div(p \vee q)=\emptyset$. Let $\sim$ be the operator of shielded base contraction induced by $\div$ and a set $R$.

(a) If $R=\mathcal{L} \backslash(C n(p) \cup C n(q))$, then $R$ satisfies uniform retractability and non-retractability propagation. ${ }^{11}$ Therefore, by Definition 4.5, $\sim$ is a SPMC and a SP-SPMC. On the other hand it holds that $p \notin R, q \notin R$ and $p \wedge q \in R$. Hence $A \sim p=A \sim q=A$ but $A \sim(p \wedge q)=A \div(p \wedge q)=\{p\} \neq A$. Thus $\sim$ does not satisfy conjunctive constancy. Therefore, according to Corollary $4.6, \sim$ is not a CC-SPMC nor a SP+CC-SPMC.

(b) If $R=\mathcal{L} \backslash(C n(\emptyset) \cup(C n(p) \backslash C n(q)))$, then $R$ satisfies uniform retractability and conjunctive completeness. ${ }^{12}$

Therefore, according to Definition 4.5, $\sim$ is a SPMC and a CC-SPMC. On the other hand it holds that $p \vee q \in R$ and $p \notin R$. From the latter it follows that $A \sim p=A$, thus $A \sim p \vdash p$. It holds that $\vdash p \rightarrow(p \vee q)$ and that $A \sim(p \vee q)=A \div(p \vee q)=$ $\emptyset$. Hence $A \sim(p \vee q) \nvdash p \vee q$. Thus $\sim$ does not satisfy success propagation. Therefore, according to Corollary $4.6, \sim$ is not a SP-SPMC nor a SP+CC-SPMC.

(c) If $R=\mathcal{L} \backslash C n(q)$, then $R$ satisfies conjunctive completeness, non-retractability propagation and uniform retractability. ${ }^{13}$ Therefore, according to Definition $4.5 \sim$ is a SP+CC-SPMC (and also a SP-SPMC, a CC-SPMC and a SPMC). On the other hand it holds that $p \wedge q \in R$ and $q \notin R$. Hence $A \sim q=A \vdash q$ and $A \sim(p \wedge q)=A \div(p \wedge q) \nvdash q$. Thus $\sim$ does not satisfy persistence. Therefore, according to Corollary $4.6, \sim$ is not a P-SPMC.

\subsection{Shielded kernel base contractions}

The following representation theorem axiomatically characterizes five kinds of operators of shielded base contraction. All these operators are induced by kernel contractions but each one of them has a different type of associated set of retractable sentences. ${ }^{14}$

Theorem 4.8. Let $A$ be a belief base and $\sim$ an operator on A. Then:

\begin{tabular}{cc}
\hline $\begin{array}{c}\sim \text { is an operator of shielded base contraction induced by a } \\
\text { kernel contraction } \div \text { and a set } R \subseteq \mathcal{L} \text { that satisfies }\end{array}$ & $\begin{array}{c}\text { if and only if } \sim \text { satisfies relative success, } \\
\text { inclusion, uniformity, core-retainment and }\end{array}$ \\
\hline uniform retractability & - \\
\hline uniform retractability and non-retractability propagation & success propagation \\
\hline uniform retractability and conjunctive completeness & conjunctive constancy \\
\hline uniform retractability, non-retractability propagation and \\
conjunctive completeness
\end{tabular}

In the next definition we introduce designations for the different kinds of shielded contractions that were axiomatically characterized in the above theorem.

\footnotetext{
11 See Lemma 1 in the Appendix.

12 See Lemma 2 in the Appendix.

13 See Lemma 3 in the Appendix.

14 The definition and axiomatic characterization of smooth kernel base contractions were recalled in Definition 2.13 and Observation 2.14 .
} 
Definition 4.9. A shielded base contraction $\sim$ on a belief base $A$ induced by a kernel contraction $\div$ and a set $R \subseteq \mathcal{L}$ is a:

\begin{tabular}{cc}
\hline Designation & if and only if $R$ satisfies \\
\hline Shielded kernel contraction (SKC) & uniform retractability \\
\hline Success propagant shielded kernel contraction (SP-SKC) & uniform retr. and non-retr. propagation \\
\hline Conjunctive constant shielded kernel contraction (CC-SKC) & uniform retractability and conj. completeness \\
\hline Success propagant conjunctive constant shielded kernel & uniform retractability, non-retr. propagation \\
contraction (SP+CC-SKC) & and conjunctive completeness \\
\hline Persistent shielded kernel contraction (P-SKC) & condition $(\mathbf{R}-\div)$ \\
\hline
\end{tabular}

The following corollary follows trivially from Definition 4.9 and Theorem 4.8 .

Corollary 4.10. Let $\sim$ be an operator on A. Then:

\begin{tabular}{cc}
\hline$\sim$ is a & $\begin{array}{c}\text { if and only if } \sim \text { satisfies relative success, } \\
\text { inclusion, uniformity, core-retainment and }\end{array}$ \\
\hline$S K C$ & - \\
\hline$S P-S K C$ & success propagation \\
\hline$C C-S K C$ & conjunctive constancy \\
\hline$S P+C C-S K C$ & success propagation and conjunctive constancy \\
\hline$P-S K C$ & persistence \\
\hline
\end{tabular}

As expected the difference between the axiomatic characterizations of the classes of shielded partial meet contractions, presented in Corollary 4.6, and the axiomatic characterizations of the classes of shielded kernel contractions, presented in Corollary 4.10 , is the replacement of relevance by core-retainment.

\subsection{Shielded smooth kernel base contractions}

The following theorem presents axiomatic characterizations for five kinds of operators of shielded base contraction that are all induced by smooth kernel contractions but each one of them has a different type of associated set of retractable sentences. $^{15}$

Theorem 4.11. Let $A$ be a belief base and $\sim$ an operator on A. Then:

\begin{tabular}{cc}
\hline $\begin{array}{c}\sim \text { is an operator of shielded base contraction induced by a } \\
\text { smooth kernel contraction } \div \text { and a set } R \subseteq \mathcal{L} \text { that satisfies }\end{array}$ & $\begin{array}{c}\text { if and only if } \sim \text { satisfies relative success, } \\
\text { inclusion, uniformity, core-retainment, } \\
\text { relative closure and }\end{array}$ \\
\hline uniform retractability & - \\
\hline uniform retractability and non-retractability propagation & success propagation \\
\hline uniform retractability and conjunctive completeness & conjunctive constancy \\
\hline $\begin{array}{c}\text { uniform retractability, non-retractability propagation and } \\
\text { conjunctive completeness }\end{array}$ & success propagation and conjunctive constancy \\
\hline condition $(\mathrm{R}-\div)$ & persistence
\end{tabular}

In the following definition we introduce designations for the different kinds of shielded contractions that were axiomatically characterized in the above theorem.

Definition 4.12. A shielded base contraction $\sim$ on a belief base $A$ induced by a smooth kernel contraction $\div$ and a set $R \subseteq \mathcal{L}$ is a:

\footnotetext{
15 The definition and axiomatic characterization of smooth kernel base contractions were recalled in Definition 2.15 and Observation 2.16 .
} 


\begin{tabular}{cc}
\hline Designation & if and only if $R$ satisfies \\
\hline Shielded smooth kernel contraction (SSKC) & uniform retractability \\
\hline $\begin{array}{c}\text { Success propagant shielded smooth kernel contraction } \\
\text { (SP-SSKC) }\end{array}$ & $\begin{array}{c}\text { uniform retractability and non-retractability } \\
\text { propagation }\end{array}$ \\
\hline $\begin{array}{c}\text { Conjunctive constant shielded smooth kernel contraction } \\
\text { (CC-SSC) }\end{array}$ & $\begin{array}{c}\text { uniform retractability and conjunctive } \\
\text { completeness }\end{array}$ \\
\hline $\begin{array}{c}\text { Success propagant conjunctive constant shielded smooth } \\
\text { kernel contraction (SP+CC-SSKC) }\end{array}$ & $\begin{array}{c}\text { uniform retractability, non-retr. propagation } \\
\text { and conjunctive completeness }\end{array}$ \\
\hline Persistent shielded smooth kernel contraction (P-SSKC) & condition $(\mathbf{R}-\div)$ \\
\hline
\end{tabular}

The following corollary follows trivially from Definition 4.12 and Theorem 4.11 .

Corollary 4.13. Let $\sim$ be an operator on A. Then:

\begin{tabular}{cc}
\hline$\sim$ is a & $\begin{array}{c}\text { if and only if } \sim \text { satisfies relative success, } \\
\text { inclusion, uniformity, core-retainment, } \\
\text { relative closure and }\end{array}$ \\
\hline$S S K C$ & - \\
\hline$S P-S S K C$ & success propagation \\
\hline$C C-S S K C$ & conjunctive constancy \\
\hline$S P+C C-S S K C$ & success propagation and conjunctive constancy \\
\hline$P-S S K C$ & persistence \\
\hline
\end{tabular}

Comparing the axiomatic characterizations presented in Corollary 4.13 with the axiomatic characterizations presented in Corollary 4.10 we immediately note that, as expected, the axiomatic characterization of each one of the classes of shielded smooth kernel contractions considered can be obtained simply by adding the relative closure postulate to the set of postulates included in the axiomatic characterization of the corresponding class of shielded kernel contractions.

\subsection{Shielded basic AGM-generated base contractions}

The following representation theorem axiomatically characterizes five kinds of operators of shielded contraction. All these operators are induced by basic AGM-generated base contractions but each one of them has a different type of associated set of retractable sentences. ${ }^{16}$

Theorem 4.14. Let $A$ be a belief base and $\sim$ an operator on $A$. Then:

\begin{tabular}{cc}
\hline $\begin{array}{c}\sim \text { is an operator of shielded base contraction induced by a } \\
\text { basic AGM-generated base contraction } \div \text { and a set } R \subseteq \mathcal{L} \\
\text { that satisfies }\end{array}$ & $\begin{array}{c}\text { if and only if } \sim \text { satisfies relative success, } \\
\text { inclusion, vacuity, extensionality, disjunctive } \\
\text { elimination and }\end{array}$ \\
\hline retractability of logical equivalents & - \\
\hline non-retractability propagation & success propagation \\
\hline retr. of logical equivalents and conjunctive completeness & conjunctive constancy \\
\hline non-retractability propagation and conjunctive completeness & success propagation and conjunctive constancy \\
\hline condition $(\mathrm{R}-\div)$ & persistence \\
\hline
\end{tabular}

In the following definition we introduce designations for the different kinds of shielded contractions that were axiomatically characterized in the above theorem.

Definition 4.15. A shielded base contraction $\sim$ on a belief base $A$ induced by a basic AGM-generated base contraction $\div$ and a set $R \subseteq \mathcal{L}$ is a:

$\overline{16}$ The definition and axiomatic characterization of basic AGM-generated base contractions were recalled in Subsubsection 2.3.4. 


\begin{tabular}{cc}
\hline Designation & if and only if $R$ satisfies \\
\hline Shielded basic AGM-generated base contraction (SbAGMC) & retractability of logical equivalents \\
\hline $\begin{array}{c}\text { Success propagant shielded basic AGM-generated base } \\
\text { contraction (SP-SbAGMC) }\end{array}$ & non-retractability propagation \\
\hline $\begin{array}{c}\text { Conjunctive constant shielded basic AGM-generated base } \\
\text { contraction (CC-SbAGMC) }\end{array}$ & $\begin{array}{c}\text { retractability of logical equivalents and } \\
\text { conjunctive completeness }\end{array}$ \\
\hline $\begin{array}{c}\text { Success propagant conjunctive constant shielded basic } \\
\text { AGM-generated base contraction (SP+CC-SbAGMC) }\end{array}$ & $\begin{array}{c}\text { non-retractability propagation and } \\
\text { conjunctive completeness }\end{array}$ \\
\hline $\begin{array}{c}\text { Persistent shielded basic AGM-generated base contraction } \\
\text { (P-SbAGMC) }\end{array}$ & condition $(\mathbf{R}-\div)$ \\
\hline
\end{tabular}

It is worth to notice that the set $R$ that was used in the proof of the right-to-left part of Theorem 4.14 was $R=$ $\{\alpha: A \sim \alpha \nvdash \alpha\}$. Therefore, from Theorem 3.9, it follows that in each row of the tables presented in Theorem 4.14 and in Definition 4.15, to the list of properties of $R$ (there presented) we can add: non-retractability of tautology and non-retractability upper-bounding. We can also add retractability of logical equivalents to the list of properties of $R$ presented in rows 2, 4 and 5 . Furthermore, uniform retractability, non-retractability propagation and conjunctive completeness can be also added to the list of properties of $R$ presented in the last row. In fact, according to Observation 3.6, in the last row condition (R - $\div$ ) can even be replaced by non-retractability preservation.

The following corollary follows trivially from Definition 4.15 and Theorem 4.14.

Corollary 4.16. Let $\sim$ be an operator on A. Then:

\begin{tabular}{cc}
\hline$\sim$ is a & $\begin{array}{c}\text { if and only if } \sim \text { satisfies relative success, inclusion, } \\
\text { vacuity, extensionality, disjunctive elimination and }\end{array}$ \\
\hline$S b A G M C$ & - \\
\hline$S P-S b A G M C$ & success propagation \\
\hline$C C-S b A G M C$ & conjunctive constancy \\
\hline$S P+C C-S b A G M C$ & success propagation and conjunctive constancy \\
\hline$P-S b A G M C$ & persistence \\
\hline
\end{tabular}

Table 1 summarizes the results obtained in the representation theorems presented in this section. Given a shielded contraction $\sim$ the white cells that are on the top of the same column represent the properties that $R$ (the associated set of retractable sentences) satisfies. The white cells that are placed on the right of the same row indicate the properties that $\sim$ satisfies. Considering, for example, the class SP+CC-SSKC, by observing this table we can see that these operators satisfy success propagation, conjunctive constancy, relative closure, uniformity, core-retainment, relative success, inclusion, vacuity and extensionality. Furthermore, we can also see that a SP+CC-SSKC is a shielded contraction induced by (a smooth kernel contraction and) a set of retractable sentences that satisfies non-retractability propagation, conjunctive completeness, uniformretractability and retractability of logical equivalents.

\section{Maps between classes of shielded base contraction functions}

In this section we study the interrelations among the classes of shielded contractions introduced in the previous section. We start by presenting an observation that illustrates the interrelations among classes of shielded base contractions induced by the same type of contraction function, but each one of them with a different type of associated set of retractable sentences. Throughout this section we assume that the classes of shielded contractions mentioned are formed by operators defined on the same belief base.

Observation 5.1. Let $X$ be any one of the elements of the following set of strings: \{SPMC, SKC, SSKC, SbAGMC\}. Then:
(a) $P-X \subset S P+C C-X$.
(b) $S P+C C-X \subset C C-X$.
(c) $S P+C C-X \subset S P-X$.
(d) $C C-X \nsubseteq S P-X$ and $S P-X \nsubseteq C C-X$.
(e) $C C-X \subset X$
(f) $S P-X \subset X$.

In Fig. 2 we present a diagram that summarizes the results established in the above observation. In that diagram an arrow between two boxes symbolizes that the class of shielded contractions at the origin of the arrow is a strict subclass 
Table 1

Schematic representation of the main postulates satisfied by each one of the twenty classes of shielded contraction considered and also of the properties satisfied by the set of retractable sentences by which each of those operators is induced.

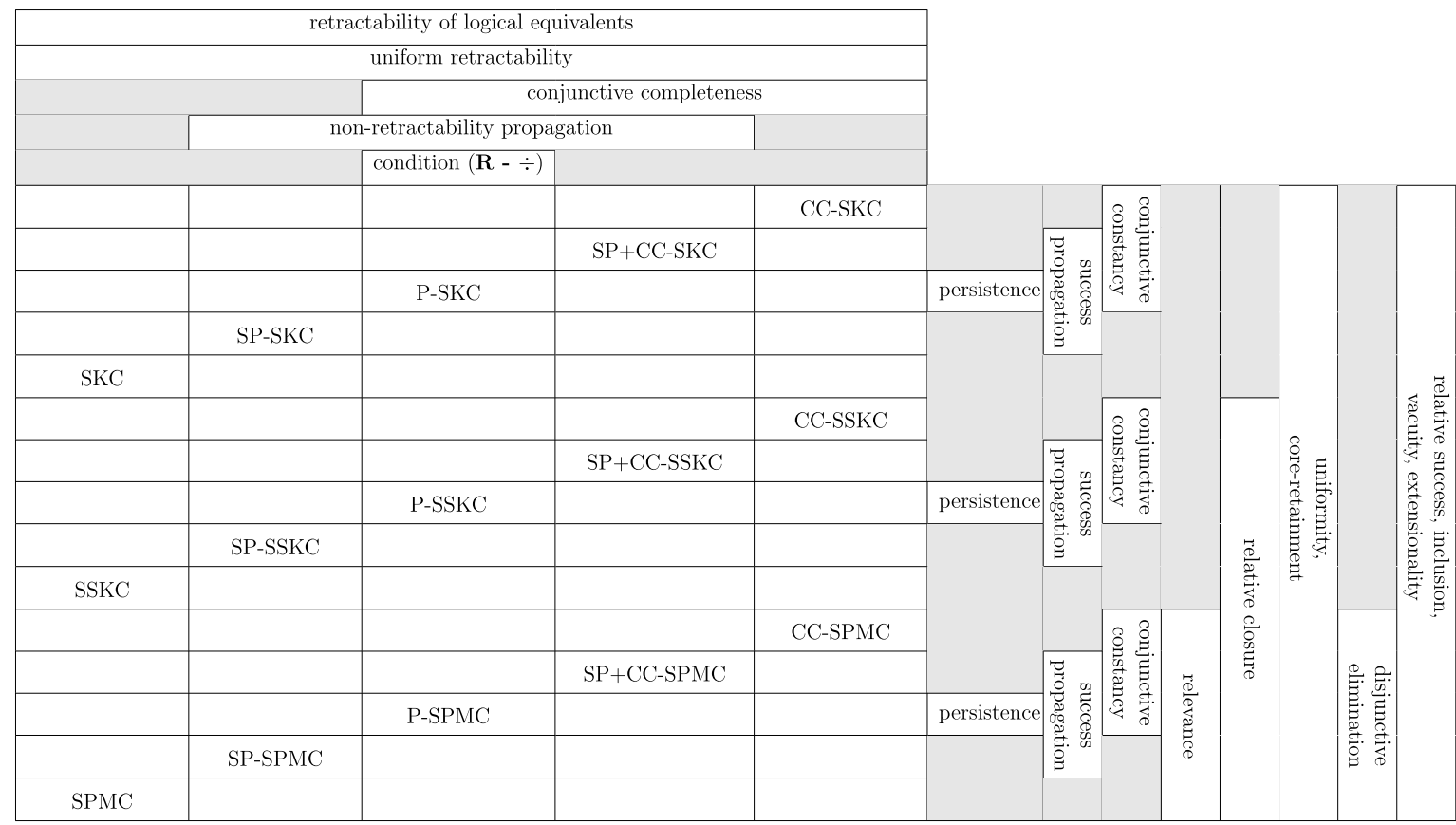

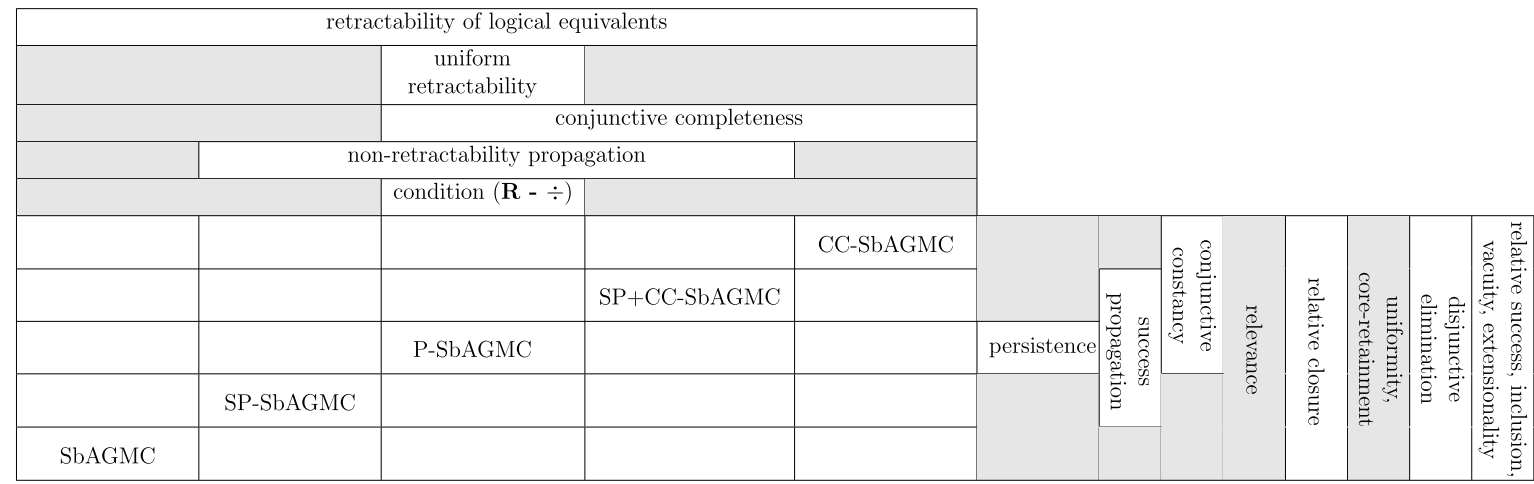

of the class of shielded contractions at the end of that arrow. The $X$ in this diagram must be replaced by an element of the following set of strings \{SPMC, SKC, SSKC, SbAGMC\}.

In what follows we will study the interrelations among classes of shielded contractions induced by different kinds of contractions. We start by presenting an example that shows that P-SSKC $\nsubseteq$ SPMC and that P-SKC $\nsubseteq$ SSKC. ${ }^{17}$

Example 5.2 (Adapted from [9, Example 22]). Let $A=\{p, p \vee q, p \leftrightarrow q, r\}$ and $R=\mathcal{L} \backslash C n(\emptyset)$, where $C n$ is purely truth-functional. It holds that $A \Perp(p \wedge q)=\{\{p, p \leftrightarrow q\},\{p \vee q, p \leftrightarrow q\}\}$.

(a) Let $\div$ be a smooth kernel contraction based on a smooth incision function $\sigma_{1}$ such that $\sigma_{1}(A \Perp(p \wedge q))=\{p, p \leftrightarrow q\}$. Let $\sim$ be the shielded base contraction induced by $\div$ and $R$. It holds that $R$ and $\div$ satisfy condition $(\mathrm{R}-\div)$. Thus, by Definition 4.12, $\sim$ is a P-SSKC. On the other hand, $p \wedge q \in R$. Thus $A \sim(p \wedge q)=A \div(p \wedge q)=\{p \vee q, r\}$. Therefore $p \notin A \sim(p \wedge q)$. Thus $\sim$ does not satisfy relevance. Hence, according to Corollary $4.6, \sim$ is not a SPMC.

17 Note that having in mind Observation 5.1 it follows from P-SSKC $\nsubseteq$ SPMC that SSKC $\nsubseteq$ SPMC, SP-SSKC $\nsubseteq$ SP-SPMC, CC-SSKC $\nsubseteq$ CC-SPMC, SP+CC-SSKC $\nsubseteq$ SP+CC-SPMC and P-SSKC $\nsubseteq$ P-SPMC. Analogously, from Observation 5.1, it follows from P-SKC $\nsubseteq$ SSKC that SKC $\nsubseteq$ SSKC, SP-SKC $\nsubseteq$ SP-SSKC, CC-SKC $\nsubseteq$ CC-SSKC, SP+CC-SKC $\nsubseteq$ SP + CC-SSKC and P-SKC $\nsubseteq$ P-SSKC. 


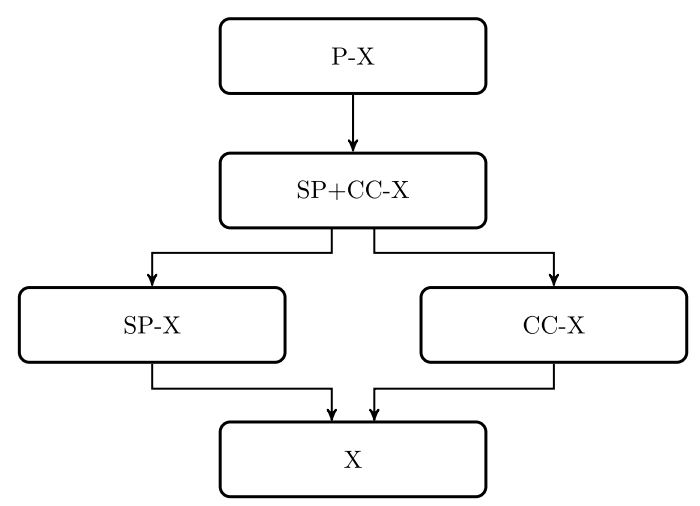

Fig. 2. Map among different classes of shielded base contraction functions induced by the same kind of contractions. The $X$ must be replaced by one of the following strings SPMC, SKC, SSKC, SbAGMC.

(b) Let $\div$ be a kernel contraction based on an incision function $\sigma_{2}$ such that $\sigma_{2}(A \Perp(p \wedge q))=\{p \vee q, p \leftrightarrow q\}$. Hence $A \div(p \wedge q)=\{p, r\}$. Let $\sim$ be the shielded base contraction induced by $\div$ and $R$. It holds that $R$ and $\div$ satisfy condition $(\mathbf{R}-\div)$. Thus, by Definition $4.9, \sim$ is a P-SKC.

On the other hand, $p \wedge q \in R$. Thus $A \sim(p \wedge q)=A \div(p \wedge q)=\{p, r\}$. Therefore $p \vee q \in A \cap C n(A \sim(p \wedge q))$ but $p \vee q \notin A \sim(p \wedge q)$. Therefore $\sim$ does not satisfy relative closure. Thus, by Corollary $4.13, \sim$ is not a SSKC.

Combining the above example with Observations 2.19 and 5.1 (see Footnote 17) we can immediately conclude that each one of the classes of SSKCs that we have considered in the previous section, on the one hand, is subsumed by the corresponding class of SKCs and, on the other hand, contains the corresponding class of SPMCs. These facts are formally stated in the following observation.

\section{Observation 5.3.}

(a) $S P M C \subset S S K C \subset S K C$.

(b) $S P-S P M C \subset S P-S S K C \subset S P-S K C$.

(c) $C C-S P M C \subset C C-S S K C \subset C C-S K C$.

(d) $S P+C C-S P M C \subset S P+C C-S S K C \subset S P+C C-S K C$.

(e) $P$-SPMC $\subset P-S S K C \subset P-S K C$.

The following example provides a shielded contraction that is a P-SbAGMC but not a SPMC nor a SKC. Therefore this example shows that P-SbAGMC $\nsubseteq$ SPMC and that P-SbAGMC $\nsubseteq$ SKC.

Example 5.4. Let $A=\{p, q, r\}$ and $C n$ be purely truth-functional. It holds that $C n(p \wedge(q \leftrightarrow r)) \in C n(A) \perp(p \wedge q)$. Let - be a partial meet contraction on $C n(A)$ such that $C n(A)-(p \wedge q)=C n(p \wedge(q \leftrightarrow r))$ and let $\div$ be an operator on $A$ defined for all $\alpha \in \mathcal{L}$ by $A \div \alpha=(C n(A)-\alpha) \cap A$. Hence $\div$ is a basic AGM-generated base contraction (since every partial meet contraction on a belief set is a basic AGM contraction [1]). On the other hand, it holds that $A \div(p \wedge q)=C n(p \wedge(q \leftrightarrow r)) \cap A=\{p\}$. Consider the set $R=\mathcal{L} \backslash C n(\emptyset)$ and let $\sim$ be the shielded base contraction induced by $\div$ and $R$. It holds that $R$ and $\div$ satisfy condition $(\mathbf{R}-\div)$. Thus, by Definition 4.15, $\sim$ is a P-SbAGMC. On the other hand $p \wedge q \in R$. Thus $r \notin A \sim(p \wedge q)=A \div(p \wedge q)$. Therefore $\sim$ does not satisfy core-retainment nor relevance. Hence, according to Corollaries 4.6 and 4.10 , $\sim$ is not a SPMC nor a SKC.

Combining the above example with Observations 2.19 and $5.1,{ }^{18}$ we can conclude that each one of the classes of SPMCs that we have considered in the previous section is strictly contained in the corresponding class of SbAGMCs. These facts are formally stated in the following observation.

\section{Observation 5.5 .}

(a) $S P M C \subset S b A G M C$.

(b) $S P-S P M C \subset S P-S b A G M C$.

\footnotetext{
18 Note that having in mind Observation 5.1 it follows from P-SbAGMC $\nsubseteq$ SPMC that SbAGMC $\nsubseteq$ SPMC, SP-SbAGMC $\nsubseteq$ SP-SPMC, CC-SbAGMC $\nsubseteq$ CC-SPMC, $\mathrm{SP}+\mathrm{CC}-\mathrm{SbAGMC} \nsubseteq \mathrm{SP}+\mathrm{CC}-\mathrm{SPMC}$ and P-SbAGMC $\nsubseteq \mathrm{P}$-SPMC.
} 


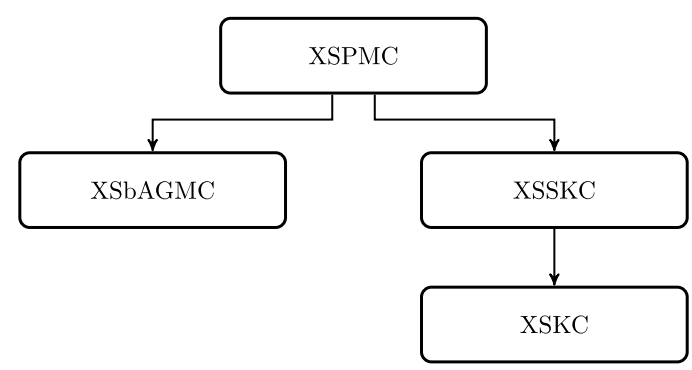

Fig. 3. Map among different kinds of shielded base contraction functions. The $\mathrm{X}$ must be replaced either by a blank space or by one of the following strings: SP-, CC-, SP+CC- or P-.

(c) CC-SPMC $\subset$ CC-SbAGMC.

(d) $S P+C C-S P M C \subset S P+C C-S b A G M C$.

(e) $P$-SPMC $\subset$ P-SbAGMC.

In the following example we present a shielded contraction that is a P-SSKC (and consequently a P-SKC) but not a SBAGMC.

Example 5.6. Let $A=\{p, p \vee q, p \rightarrow q\}$ and $C n$ be purely truth-functional. It holds that $A \Perp q=\{\{p, p \rightarrow q\},\{p \vee q, p \rightarrow q\}\}$. Let $\div$ be a smooth kernel contraction based on a smooth incision function $\sigma$ such that: $\sigma(A \Perp q)=\{p, p \rightarrow q\}$. Hence $A \div q=\{p \vee q\}$. Consider the set $R=\mathcal{L} \backslash C n(\emptyset)$ and let $\sim$ be the shielded base contraction induced by $\div$ and $R$. It holds that $R$ and $\div$ satisfy condition $(\mathbf{R}-\div)$.

Thus, by Definition 4.12, $\sim$ is a P-SSKC. On the other hand, $q \in R$. Thus $A \sim q=A \div q=\{p \vee q\}$, from which it follows that $\sim$ does not satisfy disjunctive elimination (since $p \in A \backslash A \sim q$ and $A \sim q \vdash p \vee q$ ). Therefore, by Corollary 4.16, $\sim$ is not a SbAGMC.

According to Examples 5.4 and 5.6 it holds, respectively, that P-SbAGMC $\nsubseteq$ SKC and that P-SSKC $\nsubseteq$ SbAGMC. Therefore it follows from Observations 5.1 and 5.3 that each one of the classes of SbAGMCs is not related, in terms of inclusion, neither with the corresponding class of SSKCs nor with the corresponding class of SKCs. This fact is explicitly stated in the following observation.

\section{Observation 5.7.}

(a) SKC $\nsubseteq$ SbAGMC, SbAGMC $\nsubseteq S K C$, SSKC $\nsubseteq$ SbAGMC and SbAGMC $\nsubseteq$ SSKC.

(b) SP-SKC $\nsubseteq$ SP-SbAGMC, SP-SbAGMC $\nsubseteq$ SP-SKC, SP-SSKC $\nsubseteq$ SP-SbAGMC and SP-SbAGMC $\nsubseteq$ SP-SSKC.

(c) CC-SKC $\nsubseteq C C$-SbAGMC, CC-SbAGMC $\nsubseteq C C$-SKC, CC-SSKC $\nsubseteq C C$-SbAGMC and CC-SbAGMC $\nsubseteq C C$-SSKC.

(d) $S P+C C-S K C \nsubseteq S P+C C$-SbAGMC, SP+CC-SbAGMC $\nsubseteq S P+C C-S K C, S P+C C-S S K C \nsubseteq S P+C C-S b A G M C$ and SP+CC-SbAGMC $\nsubseteq$ $S P+C C-S S K C$

(e) $P$-SKC $\nsubseteq P$-SbAGMC, P-SbAGMC $\nsubseteq P$-SKC, P-SSKC $\nsubseteq P$-SbAGMC and P-SbAGMC $\nsubseteq P$-SSKC.

In Fig. 3 we present a diagram that summarizes the results presented in this section. The $\mathrm{X}$ in that diagram is either a blank space or an element of the following set of strings: $\{$ SP-, CC-, SP+CC-, P- $\}$.

\section{Related works}

In Section 2 we have recalled several classes of non-prioritized contractions, namely shielded contractions for belief sets and for belief bases. Most of the remaining work on non-prioritized belief change presented in the literature deals with revision (rather than contraction). In this section we shall present a brief survey of existing works on non-prioritized belief revision. ${ }^{19}$

Makinson proposed in [26] an operator of non-prioritized belief revision designated by screened revision. In this model a set $A$ of sentences that are immune to revision is considered and a revision of a belief set $\mathbf{K}$ by a given sentence $\alpha$ only gives rise to a new (appropriately changed) belief set if the input sentence $\alpha$ is consistent with $A \cap \mathbf{K}$ (otherwise the belief set $\mathbf{K}$ is left unchanged).

A more general approach was proposed by Hansson in [22], by replacing $A$ by a function $f$ that is applied to the (input) sentence by which the belief set is revised. This proposal was called generalized screened revision. Several properties were proposed for $f$. For example, $f(\alpha)=\{\beta: \alpha<\beta\}$, where $<$ is a binary relation on $\mathcal{L}$.

$\overline{19}$ For a more complete overview on non-prioritized belief revision see [23,8]. 
Credibility-limited revision [25] is another operator of non-prioritized revision. When revising a belief set by a sentence, it is first analysed whether that sentence is credible or not. When revising by a credible sentence, the operator works as an AGM revision operator, otherwise it leaves the original belief set unchanged. This construction can be further specified by adding constrains to the structure of $C$ (the set of credible sentences). In [25] several properties that are natural to be expected to be satisfied by such a set were proposed and several representation theorems were presented. Also in [25] this model was developed in terms of epistemic entrenchment and possible world models. Later, in [10], the credibility-limited revision operators were extended to the belief base context and in [4] to iterated revision.

In [5] Fermé and Hansson proposed an operator that, instead of accepting the new information or rejecting it completely, allows the acceptance of only part of the new information and the rejection of the rest of it. They called this operator selective revision. An operator $\odot$ of selective revision is constructed from a partial meet revision operator $\star$ and a transformation function $f$ from $\mathcal{L}$ to $\mathcal{L}$ as follows: $\mathbf{K} \odot \alpha=\mathbf{K} \star f(\alpha)$. Intuitively, $f$ selects the credible part of every sentence. A natural restriction is that $f(\alpha)$ should not contain more information that the one that is contained in $\alpha(i . e$., $\vdash \alpha \rightarrow f(\alpha))$. In [5] several other plausible properties that a transformation function may be expected to satisfy were proposed and some representation theorems were presented.

Consolidation was introduced in [17] and consists of making an inconsistent belief base consistent. It can be seen as a contraction by $\perp$ (falsum). Consolidation is an operator for belief bases and does not have a plausible counterpart for belief sets. This is due to the fact that there is only a single inconsistent belief set and once it is obtained all distinctions are lost and consolidation can not restore them.

Hansson in [22] proposed a modification of external revision that he designated by semi-revision. Instead of contracting by the negation of a sentence, the expanded set is contracted by $\perp$ (falsum). Thus, the semi-revision can be seen as the result of the consolidation of an expansion. We note that in a semi-revision process the input sentence may be removed during the consolidation. Thus semi-revision is a non-prioritized change operator. In [17,22], Hansson defined and axiomatically characterized operators of kernel and partial-meet consolidation and of kernel and partial meet semi-revision, based in the namesake operators of contraction and revision.

\section{Conclusion}

Standard contraction operators are always successful, i.e. they are such that the result of contracting a (non-tautological) belief from a given belief base is a new belief base which (is contained in the original one and) does not imply that belief. However, as discussed before, this is not a realistic feature of belief contraction. An agent may have a set of beliefs (not necessarily tautologies) that he/she is not willing to give up independently of the contraction to be performed. The basic idea of shielded contraction is to define a function in two steps. The first step consists of determining which beliefs are suitable to be contracted and which are not, i.e. given a belief, first it is analysed if it belongs to the set of retractable sentences (set of beliefs suitable to be contracted) or not. Afterwards the function should:

- leave the set of beliefs unchanged when the belief to be contracted is considered irretractable;

- behave as a standard contraction when contracting by a retractable belief.

This kind of operators is useful for modelling the behaviour of a rational agent when it receives some new information that forces the disbelief in one of its current beliefs. In a context where the belief states of an agent are represented by belief bases, a shielded base contraction can be used to obtain the new belief state of the agent, after such an information is received. We notice that by means of this kind of operators it is possible to obtain more realistic models than those that can be obtained using (only) standard contractions, since it is naturally expectable that a rational agent will not always be willing to give up any of its present beliefs even if some external new information compels it to do so.

The present paper constitutes a thorough study of shielded contractions on belief bases. In Section 3 we presented several results highlighting some direct relations among the postulates satisfied by a shielded contraction function $\sim$ induced by a standard contraction $\div$ and a set of retractable sentences $R$ and the postulates satisfied by $\div$ and the properties of the set $R$. From the conclusions that can be drawn from those results we highlight the two following ones, concerning the postulates of relative success and persistence:

(i) The shielded base contraction $\sim$ satisfies relative success if and only if $\div$ satisfies failure or the set $R$ satisfies nonretractability of tautology.

(ii) If $R$ and $\div$ satisfy condition $(\mathbf{R}-\div)$ then $\sim$ satisfies persistence. Furthermore if $R=\{\alpha: A \sim \alpha \nvdash \alpha\}$ and $\sim$ satisfies persistence then $R$ and $\div$ satisfy condition $(\mathrm{R}-\div)$.

We are giving special attention here to the postulates of relative success and persistence since these two postulates can be considered to be the most characteristic (and intuitive) properties of shielded contraction (together with inclusion). On the one hand relative success can be thought of as the most natural weakened version of the success postulate that complies with the idea underlying the notion of shielded contraction. On the other hand, among the postulates here considered to characterize shielded contractions, persistence can be seen as the one that more accurately describes the behaviour that is expected from a shielded contraction. 
In this paper we have considered that a reasonable shielded contraction operator should satisfy at least the postulates of inclusion and relative success, and we presented a representation theorem-which is essentially based on the result mentioned in (i) above-for the class of (shielded contraction) operators that satisfy exactly those two postulates. In what concerns persistence, according to (ii) above, in order to assure that the shielded contraction, that is built from a contraction $\div$ and a set $R$, satisfies that postulate, it is enough to impose that $R$ and $\div$ satisfy condition $(\mathbf{R}-\div)$.

Sections 4 and 5 contain the other contributions of this paper, namely the proposal and axiomatic characterization of twenty kinds of shielded contractions and the study of the interrelations among all those classes in terms of inclusion. All the shielded contractions considered are defined by means of a base contraction function (of some well known class of such operators) and a certain set $R$ formed by the so-called retractable sentences. Among these classes there are four which are formed by shielded contraction operators that satisfy the above highlighted postulate of persistence.

By means of the provided results it is possible to predict the behaviour of any of the functions constructed as indicated in each of the definitions presented. On the other hand, it is also possible to use these results in the converse direction, that is, for certain sets of properties that are desirable from a shielded contraction function, our results allow to identify an explicit construction of a function that will satisfy all the properties included in that set.

\section{Acknowledgements}

We wish to thank the editors and the three anonymous referees of the Artificial Intelligence journal for their very perceptive and pertinent comments on the previous version of the paper that led to a substantial improvement. M.G. and M.R. were supported by FCT-Fundação para a Ciência e a Tecnologia-through project UID/MAT/04674/2013 (CIMA). E.F. was supported by FCT through project UID/CEC/04516/2013 (NOVA LINCS) and grant SFRH/BSAB/127790/2016 and by FAPESP 2016/13354-3.

\section{Appendix. Proofs}

Lemma 1. Let $A=\{p, q\}$ and $R=\mathcal{L} \backslash(C n(p) \cup C n(q))$. Then $R$ satisfies uniform retractability and non-retractability propagation.

Proof. Uniform retractability: Assume that it holds for all subsets $A^{\prime}$ of $A$ that $\alpha \in C n\left(A^{\prime}\right)$ if and only if $\beta \in C n\left(A^{\prime}\right)$. We will prove that $\alpha \in R$ if and only if $\beta \in R$.

Let $\alpha \notin R$. Then $\alpha \in C n(p)$ or $\alpha \in C n(q)$. Therefore $\beta \in C n(p)$ or $\beta \in C n(q)$. In both cases it follows that $\beta \notin R$. By symmetry of the case it follows that if $\beta \notin R$, then $\alpha \notin R$. Therefore $\alpha \in R$ if and only if $\beta \in R$.

Non-retractability propagation: Let $\alpha \notin R$ and $\beta \in C n(\alpha)$. From $\alpha \notin R$ it follows that $\alpha \in C n(p)$ or $\alpha \in C n(q)$. Hence $\beta \in C n(p)$ or $\beta \in C n(q)$. In both cases it follows that $\beta \notin R$. Thus $R$ satisfies non-retractability propagation.

Lemma 2. Let $A=\{p, q\}$ and $R=\mathcal{L} \backslash(C n(\emptyset) \cup(C n(p) \backslash C n(q)))$. Then $R$ satisfies uniform retractability and conjunctive completeness.

Proof. Uniform retractability: Assume that it holds for all subsets $A^{\prime}$ of $A$ that $\alpha \in C n\left(A^{\prime}\right)$ if and only if $\beta \in C n\left(A^{\prime}\right)$. We will prove that $\alpha \in R$ if and only if $\beta \in R$.

Let $\alpha \notin R$. Then $\alpha \in C n(\emptyset)$ or $\alpha \in C n(p) \backslash C n(q)$. In the first case it follows that $\beta \in C n(\emptyset)$. Therefore $\beta \notin R$. Assume now that $\alpha \in C n(p) \backslash C n(q)$. Therefore $\alpha \in C n(p)$ and $\alpha \notin C n(q)$. Hence $\beta \in C n(p)$ and $\beta \notin C n(q)$. Hence $\beta \notin R$. By symmetry of the case it follows that if $\beta \notin R$, then $\alpha \notin R$. Therefore $\alpha \in R$ if and only if $\beta \in R$.

Conjunctive completeness: Let $\alpha \wedge \beta \in R$. Thus $\alpha \wedge \beta \notin C n(\emptyset)$ and $\alpha \wedge \beta \notin C n(p) \backslash C n(q)$. Hence $\alpha \wedge \beta \notin C n(p)$ or $\alpha \wedge \beta \in C n(q)$. In the former case $\alpha \notin C n(p)$ or $\beta \notin C n(p)$. Hence $\alpha \in R$ or $\beta \in R$. In the latter case, $\alpha \in C n(q)$ and $\beta \in C n(q)$. Since $\alpha \notin C n(\varnothing)$ or $\beta \notin C n(\emptyset)$ (because $\alpha \wedge \beta \notin C n(\emptyset)$ ), it follows that $\alpha \in R$ or $\beta \in R$. Therefore $R$ satisfies conjunctive completeness.

Lemma 3. Let $A=\{p, q\}$ and $R=\mathcal{L} \backslash C n(q)$. Then $R$ satisfies conjunctive completeness, non-retractability propagation and uniform retractability.

Proof. Conjunctive completeness: Let $\alpha \wedge \beta \in R$. Hence $\{q\} \nvdash \alpha \wedge \beta$. Therefore $\{q\} \nvdash \alpha$ or $\{q\} \nvdash \beta$, from which it follows that $\alpha \in R$ or $\beta \in R$.

Non-retractability propagation: Let $\alpha \notin R$. Then $\{q\} \vdash \alpha$. Let $\beta \in C n(\alpha)$. Hence $\{q\} \vdash \beta$, from which it follows that $\beta \notin R$.

Uniform retractability: Assume that it holds for all subsets $A^{\prime}$ of $A$ that $\alpha \in C n\left(A^{\prime}\right)$ if and only if $\beta \in C n\left(A^{\prime}\right)$. Let $\alpha \notin R$. Hence $\{q\} \vdash \alpha$, from which it follows that $\{q\} \vdash \beta$. Thus $\beta \notin R$. It also holds, by symmetry of the case, that if $\beta \notin R$, then $\alpha \notin R$. Hence $\alpha \in R$ if and only if $\beta \in R$. 


\section{Proof of Observation 2.22 .}

(a) Let $A$ be a belief base and $\sim$ an operator on $A$ that satisfies relative success. Let $\alpha \in \mathcal{L}$ be such that $\vdash \alpha$. By relative success it follows that $A \sim \alpha=A$ or $A \sim \alpha \nvdash \alpha$. The latter does not hold since $\alpha$ is a tautology. Hence $A \sim \alpha=A$.

(b) Assume that $A \sim \alpha=A \sim \beta=A$. If $A \vdash \alpha$ and $A \vdash \beta$, then $A \sim \alpha \vdash \alpha$ and $A \sim \beta \vdash \beta$. Hence, by persistence, $A \sim$ $(\alpha \wedge \beta) \vdash \alpha$ and $A \sim(\alpha \wedge \beta) \vdash \beta$, from which it follows that $A \sim(\alpha \wedge \beta) \vdash \alpha \wedge \beta$. Therefore by relative success it follows that $A \sim(\alpha \wedge \beta)=A$.

If $A \nvdash \alpha$ or $A \nvdash \beta$ it follows that $A \nvdash \alpha \wedge \beta$. From which it follows, by inclusion and vacuity, that $A \sim(\alpha \wedge \beta)=A$.

Proof of Observation 3.3. Let $R$ be a set that satisfies non-retractability of tautology, non-retractability propagation and conjunctive completeness. We intend to prove that: $\alpha \in \mathcal{L} \backslash R$ if and only if $\mathcal{L} \backslash R \vdash \alpha$.

Left to right is trivial. For the other direction consider that $\mathcal{L} \backslash R \vdash \alpha$. If $\vdash \alpha$, then $\alpha \notin R$ by non-retractability of tautology. Assume now that $\nvdash \alpha$. Hence by compactness there is a non-empty finite set $H=\left\{\gamma_{1}, \ldots, \gamma_{n}\right\}$ such that $H \subseteq \mathcal{L} \backslash R$ and $H \vdash \alpha$. Hence $\left\{\gamma_{1} \wedge \ldots \wedge \gamma_{n}\right\} \vdash \alpha$. By repeated use of conjunctive completeness it follows that $\gamma_{1} \wedge \ldots \wedge \gamma_{n} \notin R$. Hence, by non-retractability propagation, $\alpha \notin R$.

\section{Proof of Observation 3.4.}

(a) Let $A$ be a set of sentences and assume $R$ satisfies uniform retractability with respect to $A$. Let $\alpha, \beta$ be such that $\vdash \alpha \leftrightarrow \beta$. Hence, for any set $A, \alpha$ and $\beta$ are implied by exactly the same subsets of $A$. Thus, by uniform retractability, $\alpha \in R$ if and only if $\beta \in R$.

(b) Retractability of logical equivalents: Let $\alpha, \beta$ be such that $\vdash \alpha \leftrightarrow \beta$. Hence $\alpha \in C n(\beta)$ and $\beta \in C n(\alpha)$. If $\alpha \notin R$, then by non-retractability propagation $\beta \notin R$. It also holds that if $\beta \notin R$, then $\alpha \notin R$. Therefore $\alpha \in R$ if and only if $\beta \in R$.

Converse conjunctive completeness: Let $\alpha \in R$ and assume by reductio ad absurdum that $\alpha \wedge \beta \notin R$. From the latter, by non-retractability propagation, it follows that $\operatorname{Cn}(\alpha \wedge \beta) \cap R=\emptyset$. Hence $\alpha \notin R$ which is a contradiction. Therefore $\alpha \wedge \beta \in R$.

Proof of Theorem 3.5. Let $\sim$ be an operator of shielded base contraction induced by a contraction operator $\div$ for $A$ and a set $R \subseteq \mathcal{L}$. We intend to prove that $R$ satisfies non-retractability of tautology and non-retractability upper bounding if and only if $R=\{\alpha: A \sim \alpha \nvdash \alpha\}$.

(Left to right)

Let $\beta \in R$. Hence, $A \sim \beta=A \div \beta$. Furthermore, by non-retractability of tautology, $\nvdash \beta$. Therefore, by $\div$ success, $A \sim \beta \nvdash \beta$.

Let $\beta \notin R$. Hence, $A \sim \beta=A$ and by non-retractability upper bounding $A \vdash \beta$. Therefore $A \sim \beta \vdash \beta$.

Hence $R=\{\alpha: A \sim \alpha \nvdash \alpha\}$.

(Right to left)

Let $R=\{\alpha: A \sim \alpha \nvdash \alpha\}$. From the definition of $R$ it follows trivially that $R$ satisfies non-retractability of tautology. Let $\beta \in \mathcal{L} \backslash R$. Hence $A \sim \beta \vdash \beta$ and $A \sim \beta=A$. Therefore $A \vdash \beta$. Hence $R$ satisfies non-retractability upper bounding.

Proof of Observation 3.6. Let $A$ be a belief base, $R \subseteq \mathcal{L}$ and $\sim$ be a shielded base contraction induced by a contraction operator $\div$ and $R$. We intend to prove that $R$ satisfies non-retractability preservation if and only if $R$ satisfies non-retractability upper bounding, and $\div$ and $R$ satisfy condition $(\mathbf{R}-\div)$.

Assume first that $R$ satisfies non-retractability preservation.

Condition ( $\mathbf{R}-\div$ ): Assume that $\alpha \notin R$ and $\beta \in R$. By non-retractability preservation it follows that $A \sim \beta \vdash \alpha$. On the other hand $A \sim \beta=A \div \beta$, by definition of $\sim$. Therefore $A \div \beta \vdash \alpha$.

Non-retractability upper bounding: By non-retractability preservation $\mathcal{L} \backslash R \subseteq C n(A \sim \alpha)$. On the other hand, by definition of shielded base contraction, $A \sim \alpha \subseteq A$. Hence, by monotony, $\mathcal{L} \backslash R \subseteq \operatorname{Cn}(A)$.

Assume now that $R$ satisfies non-retractability upper bounding and that $\div$ and $R$ are related through condition ( $\mathbf{R}-\div$ ). Let $\alpha \in \mathcal{L} \backslash R$ and $\beta \in \mathcal{L}$. We intend to prove that $A \sim \beta \vdash \alpha$.

If $\beta \in R$, then it follows by definition of $\sim$ that $A \sim \beta=A \div \beta$. On the other hand, by condition ( $\mathbf{R}-\div)$ it follows that $A \div \beta \vdash \alpha$. Hence $A \sim \beta \vdash \alpha$.

Assume now that $\beta \notin R$. Hence, by definition of $\sim$, it follows that $A \sim \beta=A$. On the other hand by non-retractability upper bounding it follows that $A \vdash \alpha$. Thus $A \sim \beta \vdash \alpha$.

Proof of Observation 3.7. Let $A$ be a belief base, $\div$ be a contraction on $A, R \subseteq \mathcal{L}$, and $\sim$ be the shielded base contraction induced by $\div$ and $R$. Assume that $R$ satisfies non-retractability preservation and non-retractability of tautology.

Conjunctive completeness: Let $\alpha \wedge \beta \in R$ and assume by reductio ad absurdum that $\alpha \notin R$ and $\beta \notin R$. By non-retractability of tautology $\nvdash \alpha \wedge \beta$. On the other hand, by non-retractability preservation $\mathcal{L} \backslash R \subseteq C n(A \sim(\alpha \wedge \beta))$. Hence $A \sim(\alpha \wedge \beta) \vdash \alpha$ and $A \sim(\alpha \wedge \beta) \vdash \beta$. Therefore $A \sim(\alpha \wedge \beta) \vdash \alpha \wedge \beta$. By definition of $\sim A \sim(\alpha \wedge \beta)=A \div(\alpha \wedge \beta)$. Thus $A \div(\alpha \wedge \beta) \vdash \alpha \wedge \beta$, which contradicts $\div$ success. 
Non-retractability propagation: Let $\alpha \notin R$ and $\beta \in C n(\alpha)$. If $\vdash \beta$, then by non-retractability of tautology $\beta \notin R$. Consider now that $\nvdash \beta$. By non-retractability preservation $\mathcal{L} \backslash R \subseteq C n(A \sim \beta)$. Hence $A \sim \beta \vdash \alpha$. Therefore $A \sim \beta \vdash \beta$. Hence $\beta \notin R$ (otherwise it would follow that $A \div \beta \vdash \beta$, which contradicts $\div$ success).

Uniform retractability: Assume that for all subsets $A^{\prime}$ of $A, A^{\prime} \vdash \alpha$ if and only if $A^{\prime} \vdash \beta$. Assume that $\alpha \notin R$. By non-retractability preservation $\mathcal{L} \backslash R \subseteq C n(A \sim \beta)$. Hence $A \sim \beta \vdash \alpha$. Since $\sim$ is a shielded base contraction it follows that $A \sim \beta \subseteq A$. Hence, by hypothesis, $A \sim \beta \vdash \beta$. Assume by reductio ad absurdum that $\beta \in R$. Thus by non-retractability of tautology $\nvdash \beta$. On the other hand, by definition of $\sim, A \sim \beta=A \div \beta$. Therefore $A \div \beta \vdash \beta$, which contradicts $\div$ success. Hence if $\alpha \notin R$, then $\beta \notin R$. By symmetry of the case it holds that if $\beta \notin R$, then $\alpha \notin R$. Hence $\alpha \in R$ if and only if $\beta \in R$.

Retractability of logical equivalents: Follows by Observation 3.4 since, as shown above, $R$ satisfies uniform retractability.

Proof of Theorem 3.8. Let $A$ be a belief base, $\div$ be a contraction on $A, R \subseteq \mathcal{L}$, and $\sim$ be the shielded base contraction induced by $\div$ and $R$.

Thus,

$$
A \sim \alpha= \begin{cases}A \div \alpha & \text { if } \alpha \in R \\ A & \text { otherwise }\end{cases}
$$

where $\div$ is an operator on $A$ that satisfies success and inclusion.

(a) It follows trivially from its definition that $\sim$ satisfies inclusion.

Assume that $\div$ satisfies vacuity. It follows trivially from its definition that $\sim$ satisfies vacuity.

Assume that $\div$ satisfies failure. If $\alpha \notin R$, then $A \sim \alpha=A$. Assume now that $\alpha \in R$. Hence $A \sim \alpha=A \div \alpha$. If $\nvdash \alpha$, then it follows, by $\div$ success, that $A \sim \alpha \nvdash \alpha$. If $\vdash \alpha$, then by $\div$ failure $A \sim \alpha=A \div \alpha=A$. Therefore $\sim$ satisfies relative success. Assume that $\div$ satisfies relative closure. If $\alpha \notin R$ it follows trivially that $A \cap \operatorname{Cn}(A \sim \alpha) \subseteq A \sim \alpha$. Assume now that $\alpha \in R$. Then $A \sim \alpha=A \div \alpha$, and it follows trivially by $\div$ relative closure that $A \cap C n(A \sim \alpha) \subseteq A \sim \alpha$. Therefore $\sim$ satisfies relative closure.

Assume that $\div$ satisfies relevance. Let $\beta \in A$ and $\beta \notin A \sim \alpha$. Hence $A \sim \alpha=A \div \alpha$, and it follows trivially from $\div$ relevance that $\sim$ satisfies relevance.

Assume that $\div$ satisfies core-retainment. Let $\beta \in A$ and $\beta \notin A \sim \alpha$. Hence $A \sim \alpha=A \div \alpha$, and it follows trivially from $\div$ core-retainment that $\sim$ satisfies core-retainment.

Assume that $\div$ satisfies disjunctive elimination. Let $\beta \in A$ and $\beta \notin A \sim \alpha$. Hence $A \sim \alpha=A \div \alpha$, and it follows trivially from $\div$ disjunctive elimination that $\sim$ satisfies disjunctive elimination.

(b) Let $R$ and $\div$ be such that condition $(\mathbf{R}-\div)$ is satisfied.

Now we will show that $\sim$ satisfies persistence. Let $\alpha, \beta \in \mathcal{L}$. Assume that $A \sim \beta \vdash \beta$. We intend to prove that $A \sim \alpha \vdash \beta$. It follows trivially if $\vdash \beta$. Assume now that $\nvdash \beta$. By $\div$ success it follows that $A \div \beta \nvdash \beta$. Hence $A \sim \beta \neq A \div \beta$. Thus, by definition of $\sim$, it follows that $\beta \notin R$. Therefore $A \sim \beta=A$, from which it follows that $A \vdash \beta$. If $\alpha \in R$, then it follows by condition $(\mathbf{R}-\div)$ that $A \sim \alpha \vdash \beta$. If $\alpha \notin R$, then $A \sim \alpha=A$. Hence $A \sim \alpha \vdash \beta$.

Assume that $\div$ satisfies failure and extensionality. Let $\alpha, \beta$ be such that $\vdash \alpha \leftrightarrow \beta$. We must prove that $A \sim \alpha=A \sim \beta$. It holds that $\vdash \alpha$ if and only if $\vdash \beta$. Therefore there are two cases to consider:

Case 1) $\vdash \alpha$ and $\vdash \beta$. Hence, by $\div$ failure, $A \div \alpha=A \div \beta=A$. Then $A \sim \alpha=A \sim \beta=A$.

Case 2) $\nvdash \alpha$ and $\nvdash \beta$. Assume by reductio ad absurdum, without loss of generality, that $\alpha \notin R$ and $\beta \in R$. By condition $(\mathbf{R}-\div)$ it follows that $A \div \beta \vdash \alpha$. Thus $A \div \beta \vdash \beta$, which contradicts $\div$ success. Hence $\alpha \in R$ if and only if $\beta \in R$. We will prove by cases:

Case 2.1) $\alpha \in R$ and $\beta \in R$. Hence $A \sim \alpha=A \div \alpha$ and $A \sim \beta=A \div \beta$. Thus, by $\div$ extensionality, $A \sim \alpha=A \sim \beta$.

Case 2.2) $\alpha \notin R$ and $\beta \notin R$. Thus $A \sim \alpha=A \sim \beta=A$.

Assume that $\div$ satisfies failure and uniformity. Assume that for all subsets $A^{\prime}$ of $A, A^{\prime} \vdash \alpha$ if and only if $A^{\prime} \vdash \beta$. We must prove that $A \sim \alpha=A \sim \beta$. If $\vdash \alpha$, then $\vdash \beta$ (since $\emptyset \subseteq A$ ). By $\div$ failure, $A \div \alpha=A \div \beta=A$. Hence, by definition of $\sim$, it follows that $A \sim \alpha=A \sim \beta=A$.

Consider now that $\nvdash \alpha$. Hence $\nvdash \beta$. Assume without loss of generality, that $\alpha \notin R$ and $\beta \in R$. By condition (R - $\div$ ) it follows that $A \div \beta \vdash \alpha$. On the other hand, it follows from $\div$ inclusion that $A \div \beta \subseteq A$. Hence by hypothesis, $A \div \beta \vdash \beta$, which contradicts $\div$ success. Hence $\alpha \in R$ if and only if $\beta \in R$.

So there are two cases to consider:

Case 1) $\alpha \in R$ and $\beta \in R$. Then $A \sim \alpha=A \div \alpha$ and $A \sim \beta=A \div \beta$. By $\div$ uniformity $A \div \alpha=A \div \beta$. Hence $A \sim \alpha=A \sim \beta$. Case 2) $\alpha \notin R$ and $\beta \notin R$. Then $A \sim \alpha=A \sim \beta=A$.

(c) Let $R$ be a set that satisfies non-retractability preservation.

It follows from Observation 3.6 that $\div$ and $R$ satisfy condition ( $\mathbf{R}-\div)$. The rest of the proof follows trivially from (b).

(d) It follows trivially if $\alpha \notin R$. Assume now that $\alpha \in R$. Hence $A \sim \alpha=A \div \alpha$. By non-retractability of tautology, it follows that $\nvdash \alpha$. Thus, from $\div$ success it follows that $A \sim \alpha \nvdash \alpha$.

(e) Let $\vdash \alpha \leftrightarrow \beta$. By retractability of logical equivalents it follows that $\alpha \in R$ if and only if $\beta \in R$. Thus, there are two cases to consider: 
Case 1) $\alpha \notin R$ and $\beta \notin R$. Then $A \sim \alpha=A \sim \beta=A$.

Case 2) $\alpha \in R$ and $\beta \in R$. Then $A \sim \alpha=A \div \alpha$ and $A \sim \beta=A \div \beta$. Thus by $\div$ extensionality it follows that $A \sim \alpha=A \sim \beta$.

(f) Assume that for all subsets $A^{\prime}$ of $A, A^{\prime} \vdash \alpha$ if and only if $A^{\prime} \vdash \beta$. By uniform retractability $\alpha \in R$ if and only if $\beta \in R$. There are two cases to consider:

Case 1) $\alpha \in R$ and $\beta \in R$. Then $A \sim \alpha=A \div \alpha$ and $A \sim \beta=A \div \beta$. By $\div$ uniformity $A \div \alpha=A \div \beta$. Hence $A \sim \alpha=A \sim \beta$. Case 2) $\alpha \notin R$ and $\beta \notin R$. Then $A \sim \alpha=A \sim \beta=A$.

(g) Let $A \sim \beta \vdash \beta$ and $\vdash \beta \rightarrow \alpha$.

Case 1) $\beta \notin R$. Hence $A \sim \beta=A$ and $A \vdash \alpha$. It follows by non-retractability propagation that $\alpha \notin R$. Therefore $A \sim \alpha=A$ and $A \sim \alpha \vdash \alpha$.

Case 2) $\beta \in R$. Hence $A \sim \beta=A \div \beta$. Thus, by $\div$ success, it follows that $\vdash \beta$. Thus $\vdash \alpha$ and (consequently) $A \sim \alpha \vdash \alpha$.

Assume that $\div$ satisfies extensionality. Let $\alpha, \beta$ be such that $\vdash \alpha \leftrightarrow \beta$. Hence $\beta \in \operatorname{Cn}(\alpha)$ and $\alpha \in \operatorname{Cn}(\beta)$, from which it follows, by non-retractability propagation, that $\alpha \in R$ if and only if $\beta \in R$. Thus if $\alpha \notin R$, then $A \sim \alpha=A=A \sim \beta$. If $\alpha \in R$, then by $\div$ extensionality, $A \sim \alpha=A \div \alpha=A \div \beta=A \sim \beta$.

(h) We start by noticing that, according to Observation 3.4, if $R$ satisfies uniform retractability, then it also satisfies retractability of logical equivalents. Now assume that $\div$ satisfies vacuity and failure and let $R$ be a set that satisfies conjunctive completeness and retractability of logical equivalents. We will prove that $\sim$ satisfies conjunctive constancy. Let $A \sim \alpha=A \sim \beta=A$. If $A \nvdash \alpha \wedge \beta$, then by $\div$ inclusion and vacuity $A \sim(\alpha \wedge \beta)=A$. Consider that $A \vdash \alpha \wedge \beta$. Hence $A \vdash \alpha$ and $A \vdash \beta$. If $\alpha \wedge \beta \notin R$, then $A \sim(\alpha \wedge \beta)=A$. Assume now that $\alpha \wedge \beta \in R$. Hence, by conjunctive completeness $\alpha \in R$ or $\beta \in R$. Assume without loss of generality that $\alpha \in R$. Hence $A \div \alpha \vdash \alpha$. Thus, by $\div$ success, $\vdash \alpha$, from which it follows that $\vdash(\alpha \wedge \beta) \leftrightarrow \beta$. Therefore, by retractability of logical equivalents, $\beta \in R$. Hence $A \div \beta \vdash \beta$. Thus, by $\div$ success, $\vdash \beta$. Therefore $\vdash \alpha \wedge \beta$, from which it follows by $\div$ failure that $A \sim(\alpha \wedge \beta)=A$.

(i) We start by noticing that, according to Observation 2.6 , if $\div$ satisfies uniformity, then it also satisfies extensionality. Now assume that $\div$ satisfies vacuity and extensionality and let $R$ be a set that satisfies non-retractability propagation and conjunctive completeness. We will show that $\sim$ satisfies conjunctive constancy. Let $A \sim \alpha=A \sim \beta=A$. We will prove by cases:

Case 1) $\vdash \alpha$. Then $\vdash \beta \leftrightarrow(\alpha \wedge \beta)$. By non-retractability propagation $\alpha \wedge \beta \in R$ if and only if $\beta \in R$, from which it follows by the definition of $\sim$ and $\div$ extensionality that $A \sim(\alpha \wedge \beta)=A \sim \beta=A$.

Case 2) $\vdash \beta$. The proof is symmetrical to the one presented in the previous case.

Case 3$) \nvdash \alpha$ and $\nvdash \beta$.

Case 3.1) $\alpha \in R$. Then $A \sim \alpha=A \div \alpha$. Hence by $\div$ success $A \sim \alpha \nvdash \alpha$. Thus $A \nvdash \alpha$ (since $A \sim \alpha=A)$. Therefore, $A \nvdash \alpha \wedge \beta$, from which it follows by $\div$ vacuity and inclusion that $A \div(\alpha \wedge \beta)=A$. Thus, by definition of $\sim A \sim(\alpha \wedge \beta)=A$.

Case 3.2) $\beta \in R$. The proof is symmetrical to the one presented in the previous case.

Case 3.3) $\alpha \notin R$ and $\beta \notin R$. Then by conjunctive completeness $\alpha \wedge \beta \notin R$. Hence $A \sim(\alpha \wedge \beta)=A$.

\section{Proof of Theorem 3.9.}

(a) That $R$ satisfies non-retractability of tautology follows trivially by definition of $R$.

Assume that $\sim$ satisfies inclusion. We will show that $R$ satisfies non-retractability upper bounding. Let $\alpha \in \mathcal{L} \backslash R$. Hence $A \sim \alpha \vdash \alpha$. Thus, by $\sim$ inclusion, $A \vdash \alpha$.

Assume that $\sim$ satisfies extensionality. Consider $\alpha, \beta \in \mathcal{L}$ such that $\vdash \alpha \leftrightarrow \beta$. Then by $\sim$ extensionality $A \sim \alpha=A \sim \beta$. Let $\alpha \notin R$. Hence $A \sim \alpha \vdash \alpha$. Thus $A \sim \beta \vdash \alpha$, from which it follows that $A \sim \beta \vdash \beta$. Hence $\beta \notin R$. By symmetry of the case, if $\beta \notin R$, then $\alpha \notin R$. Hence $\alpha \in R$ if and only if $\beta \in R$. Thus $R$ satisfies retractability of logical equivalents.

Assume that $\sim$ satisfies inclusion and uniformity. We will prove that $R$ satisfies uniform retractability. Assume that for all subsets $A^{\prime}$ of $A, A^{\prime} \vdash \alpha$ if and only if $A^{\prime} \vdash \beta$. Let $\alpha \notin R$. Hence $A \sim \alpha \vdash \alpha$, from which it follows (by hypothesis and $\sim$ inclusion) that $A \sim \alpha \vdash \beta$. Thus by $\sim$ uniformity $A \sim \beta \vdash \beta$. Therefore $\beta \notin R$. It follows by symmetry of the case that if $\beta \notin R$, then $\alpha \notin R$. Therefore $\alpha \in R$ if and only if $\beta \in R$. Hence $R$ satisfies uniform retractability.

Assume that $\sim$ satisfies success propagation. We will show that $R$ satisfies non-retractability propagation. Consider $\alpha$ such that $\alpha \notin R$. It follows that $A \sim \alpha \vdash \alpha$. Consider $\beta$ such that $\beta \in C n(\alpha)$. Hence by deduction $\vdash \alpha \rightarrow \beta$. Thus, by success propagation, $A \sim \beta \vdash \beta$. Therefore $\beta \notin R$. Hence $\operatorname{Cn}(\alpha) \cap R=\emptyset$.

Assume that $\sim$ satisfies relative success and conjunctive constancy. We will show that $R$ satisfies conjunctive completeness. Let $\alpha \notin R$ and $\beta \notin R$. It follows by definition of $R$ that $A \sim \alpha \vdash \alpha$ and $A \sim \beta \vdash \beta$. Hence, by relative success, $A \sim \alpha=$ $A \sim \beta=A$. Thus, by $\sim$ conjunctive constancy $A \sim(\alpha \wedge \beta)=A$, from which it follows that $A \sim(\alpha \wedge \beta) \vdash \alpha \wedge \beta$. Therefore $\alpha \wedge \beta \notin R$.

Assume that $\sim$ that satisfies persistence. We will show that $R$ satisfies non-retractability preservation, conjunctive completeness, non-retractability propagation and retractability of logical equivalents.

Non-retractability preservation: Let $\alpha$ be an arbitrary sentence and $\beta \in \mathcal{L} \backslash R$. Hence $A \sim \beta \vdash \beta$. By persistence $A \sim$ $\alpha \vdash \beta$. Thus $\mathcal{L} \backslash R \subseteq C n(A \sim \alpha)$.

Conjunctive completeness: Let $\alpha \notin R$ and $\beta \notin R$. Hence, by definition of $R, A \sim \alpha \vdash \alpha$ and $A \sim \beta \vdash \beta$. Thus, by persistence, $A \sim(\alpha \wedge \beta) \vdash \alpha$ and $A \sim(\alpha \wedge \beta) \vdash \beta$. Therefore $A \sim(\alpha \wedge \beta) \vdash \alpha \wedge \beta$. Thus $\alpha \wedge \beta \notin R$.

Non-retractability propagation: Let $\alpha \notin R$ and $\beta \in C n(\alpha)$. Hence, by definition of $R, A \sim \alpha \vdash \alpha$. By persistence it follows that $A \sim \beta \vdash \alpha$. Therefore $A \sim \beta \vdash \beta$. Hence, by definition of $R, \beta \notin R$. 
Retractability of logical equivalents: Follows trivially by Observation 3.4, since, as shown above, $R$ satisfies nonretractability propagation.

Assume that $\sim$ satisfies inclusion and persistence. We will show that $R$ satisfies uniform retractability. Assume that for all subsets $A^{\prime}$ of $A, A^{\prime} \vdash \alpha$ if and only if $A^{\prime} \vdash \beta$. Let $\alpha \notin R$. Hence, by definition of $R, A \sim \alpha \vdash \alpha$. By persistence it follows that $A \sim \beta \vdash \alpha$ and by inclusion $A \sim \beta \subseteq A$. Therefore by hypothesis $A \sim \beta \vdash \beta$. Hence $\beta \notin R$, by definition of $R$. It follows by symmetry of the case that if $\beta \notin R$, then $\alpha \notin R$. Hence $\alpha \in R$ if and only if $\beta \in R$.

(b) Follows trivially from (a) and Observation 3.6.

Proof of Theorem 3.10. Let $\div$ be the operator on $A$ defined by:

$$
A \div \alpha= \begin{cases}A \sim \alpha & \text { if } \alpha \in R \\ A-\alpha & \text { otherwise }\end{cases}
$$

where - is an operator on $A$ that satisfies success, inclusion, uniformity and relevance and $R=\{\alpha: A \sim \alpha \nvdash \alpha\}$. In what follows we show that statements (a), (b) and (c) hold.

(a) Assume that $\sim$ satisfies relative success and inclusion. We start by showing that $\div$ satisfies success and inclusion. It follows from $\sim$ and - inclusion that $\div$ satisfies inclusion. We will now show that $\div$ satisfies success. Let $\alpha$ be such that $\nvdash \alpha$. If $\alpha \in R$, then $A \div \alpha=A \sim \alpha$ and by definition of $R, A \sim \alpha \nvdash \alpha$. Thus $A \div \alpha \nvdash \alpha$. If $\alpha \notin R$, then $A \div \alpha=A-\alpha$. Therefore, by - success it follows that $A \div \alpha \nvdash \alpha$. Hence, according to Definition 2.5 , $\div$ is a contraction operator. Finally we show that the following equality holds:

$$
A \sim \alpha= \begin{cases}A \div \alpha & \text { if } \alpha \in R \\ A & \text { otherwise }\end{cases}
$$

If $\alpha \in R$, then by definition of $\div, A \sim \alpha=A \div \alpha$. Assume now that $\alpha \notin R$. Hence $A \sim \alpha \vdash \alpha$, from which it follows, by $\sim$ relative success, that $A \sim \alpha=A$.

(b) Assume that $\sim$ satisfies vacuity. We will prove that $\div$ satisfies vacuity. Consider that $A \nvdash \alpha$. If $\alpha \in R$, then $A \div \alpha=A \sim \alpha$. By $\sim$ vacuity it follows that $A \subseteq A \div \alpha$. If $\alpha \notin R$, then $A \div \alpha=A-\alpha$. By Observation 2.6, satisfies vacuity. Hence $A \subseteq A \div \alpha$.

Assume that $\sim$ satisfies extensionality. We will prove that $\div$ satisfies extensionality. Let $\alpha$ and $\beta$ be such that $\vdash \alpha \leftrightarrow \beta$. Let $\alpha \in R$. Hence $A \sim \alpha \nvdash \alpha$. Thus, by $\sim$ extensionality, $A \sim \beta \nvdash \alpha$. From which it follows that $A \sim \beta \nvdash \beta$. Therefore $\beta \in R$. Hence $A \div \alpha=A \sim \alpha=A \sim \beta=A \div \beta$. Let $\alpha \notin R$. Hence $\beta \notin R$. Since, according to Observation 2.6, - satisfies extensionality, it follows that $A \div \alpha=A-\alpha=A-\beta=A \div \beta$.

Assume that $\sim$ satisfies failure. We will prove that $\div$ satisfies failure. Let $\vdash \alpha$. Then $\alpha \notin R$. Hence $A \div \alpha=A-\alpha$. Since, according to Observation 2.6, - satisfies failure, it follows that $A \div \alpha=A$.

Assume that $\sim$ satisfies relative closure. We will prove that $\div$ satisfies relative closure. If $\alpha \in R$, then $A \div \alpha=A \sim \alpha$. The rest of the proof for this case follows by $\sim$ relative closure. Consider now that $\alpha \notin R$. Hence $A \div \alpha=A-\alpha$. The rest of the proof follows trivially by Observation 2.6.

Assume that $\sim$ satisfies inclusion and uniformity. We will prove that $\div$ satisfies uniformity. Assume that it holds for all subsets $A^{\prime}$ of $A$ that $A^{\prime} \vdash \alpha$ if and only if $A^{\prime} \vdash \beta$. By $\sim$ uniformity it follows that $A \sim \alpha=A \sim \beta$. Consider the case that $\alpha \in R$. Hence $A \sim \alpha \nvdash \alpha$. Thus $A \sim \beta \nvdash \alpha$. By inclusion $A \sim \beta \subseteq A$. Therefore, by hypothesis, $A \sim \beta \nvdash \beta$. Hence, $\beta \in R$. Thus $A \div \alpha=A \div \beta$. Consider now that $\alpha \notin R$. Hence $\beta \notin R$. Hence $A \div \alpha=A-\alpha$ and $A \div \beta=A-\beta$, from which it follows, by - uniformity, that $A \div \alpha=A \div \beta$.

Assume that $\sim$ satisfies relevance. We will prove that $\div$ satisfies relevance. If $\alpha \in R$, then $A \div \alpha=A \sim \alpha$ and the rest of the proof for this case follows by $\sim$ relevance. Let $\alpha \notin R$. Hence $A \div \alpha=A-\alpha$ and the rest of the proof follows trivially by - relevance.

Assume that $\sim$ satisfies core-retainment. We will prove that $\div$ satisfies core-retainment. If $\alpha \in R$, then $A \div \alpha=A \sim \alpha$ and the rest of the proof for this case follows by $\sim$ core-retainment. Let $\alpha \notin R$. Hence $A \div \alpha=A-\alpha$ and the rest of the proof follows trivially by - core-retainment (Observation 2.6).

Assume that $\sim$ satisfies disjunctive elimination. We will prove that $\div$ satisfies disjunctive elimination. If $\alpha \in R$, then $A \div \alpha=A \sim \alpha$ and the rest of the proof for this case follows by $\sim$ disjunctive elimination. Let $\alpha \notin R$. Hence $A \div \alpha=A-\alpha$, and the rest of the proof follows trivially by - disjunctive elimination (Observation 2.6).

(c) Assume that $\sim$ satisfies persistence. Let $\alpha \notin R$ and $\beta \in R$. From $\alpha \notin R$ it follows that $A \sim \alpha \vdash \alpha$. Hence, by persistence it follows that $A \sim \beta \vdash \alpha$. From $\beta \in R$ it follows that $A \div \beta=A \sim \beta$. Hence $A \div \beta \vdash \alpha$.

Proof of Theorem 4.1. Let $A$ be a belief base. We will prove this theorem by showing that condition (1) is equivalent to condition (2) and to condition (3).

$(1 \rightarrow 2)$ Let $\sim$ be an operator on $A$ that satisfies relative success and inclusion. Let $R$ be the set defined by:

$$
R=\{\alpha: A \sim \alpha \nvdash \alpha\}
$$


According to Theorem 3.9 (a) $R$ satisfies non-retractability of tautology. On the other hand, it follows from Theorem 3.10 (a) that there exists an operator $\div$ such that $\sim$ is the shielded base contraction induced by $\div$ and $R$.

$(2 \rightarrow 1)$ Let $\sim$ be the operator of shielded base contraction induced by a contraction operator $\div$ and a set $R \subseteq \mathcal{L}$ that satisfies non-retractability of tautology. Hence by Theorem 3.8 (a) and (d), satisfies relative success and inclusion.

$(1 \rightarrow 3)$ Let $\sim$ be an operator on $A$ that satisfies relative success and inclusion. By Observation 2.22 (a) it follows that $\sim$ also satisfies failure. Hence, according to Theorem $3.10((\mathrm{a})$ and (b)), is a shielded base contraction induced by an operator $\div$ that satisfies failure and $R$.

$(3 \rightarrow 1)$ Let $\sim$ be an operator of shielded base contraction induced by a contraction operator $\div$ that satisfies failure and a set $R \subseteq \mathcal{L}$. By Theorem 3.8 (a), $\sim$ satisfies relative success and inclusion.

\section{Proof of Theorems 4.4, 4.8 and 4.11.}

(Right-to-left)

Let $A$ be a belief base and $\sim$ an operator that satisfies relative success, inclusion and uniformity. Let $R$ be the set defined by:

$$
R=\{\alpha: A \sim \alpha \nvdash \alpha\}
$$

It follows from Theorem 3.9 that $R$ satisfies uniform retractability.

Furthermore, from Theorem 3.9, it follows that:

- If $\sim$ satisfies success propagation, then $R$ satisfies non-retractability propagation;

- If $\sim$ satisfies conjunctive constancy, then $R$ satisfies conjunctive completeness;

From Theorem 3.10 (a) it follows that there exists a contraction operator $\div$ such that $\sim$ is the shielded base contraction induced by $\div$ and $R$. Furthermore, from Theorem 3.10 (c), if $\sim$ satisfies persistence, then $\div$ and $R$ satisfy condition ( $\mathbf{R}-\div$ ).

\section{For Theorem 4.4}

From Theorem 3.10 and Observation 2.10 it follows that $\div$ is a partial meet contraction.

\section{For Theorem 4.8}

From Theorem 3.10 and Observation 2.14 it follows that $\div$ is a kernel contraction.

\section{For Theorem 4.11}

From Theorem 3.10 and Observation 2.16 it follows that $\div$ is a smooth kernel contraction.

\section{(Left-to-right)}

Let $A$ be a belief base, $\div$ be an operator on $A$ and $R \subseteq \mathcal{L}$. Let $\sim$ be such that:

$$
A \sim \alpha= \begin{cases}A \div \alpha & \text { if } \alpha \in R \\ A & \text { otherwise }\end{cases}
$$

From Theorem 3.8 it follows that if $\div$ satisfies success, inclusion, failure, vacuity and uniformity, then:

- If $R$ satisfies uniform retractability, then $\sim$ satisfies uniformity (Theorem $3.8(\mathrm{f})$ ).

- If $R$ satisfies non-retractability propagation, then $\sim$ satisfies success propagation (Theorem $3.8(\mathrm{~g})$ ).

- If $R$ satisfies conjunctive completeness and uniform retractability, then $\sim$ satisfies conjunctive constancy (Theorem 3.8 (h)).

- If $R$ and $\div$ satisfy condition $(\mathbf{R}-\div)$, then $\sim$ satisfies persistence and uniformity (Theorem 3.8 (b)).

\section{For Theorem 4.4}

If $\div$ is a partial meet contraction operator on $A$, then from Observation $2.10 \div$ satisfies success, inclusion, uniformity and relevance. From Observation 2.6 it follows that $\div$ satisfies failure and vacuity. Hence from Theorem 3.8 (a) it holds also that $\sim$ satisfies relative success, inclusion and relevance.

\section{For Theorem 4.8}

If $\div$ is a kernel contraction operator on $A$, then from Observation $2.14 \div$ satisfies success, inclusion, uniformity and core-retainment. From Observation 2.6 it follows that $\div$ satisfies failure and vacuity. Hence from Theorem 3.8 (a) it holds also that $\sim$ satisfies relative success, inclusion and core-retainment.

\section{For Theorem 4.11}

If $\div$ is a smooth kernel contraction operator on $A$, then from Observation $2.16 \div$ satisfies success, inclusion, uniformity, core-retainment and relative closure. From Observation 2.6 it follows that $\div$ satisfies failure and vacuity. Hence from Theorem 3.8 (a) it holds also that $\sim$ satisfies relative success, inclusion, core-retainment and relative closure. 


\section{Proof of Theorem 4.14.}

(Right-to-left)

Let $A$ be a belief base and $\sim$ an operator that satisfies relative success, inclusion, vacuity, extensionality and disjunctive elimination. Let $R$ be the set defined by:

$$
R=\{\alpha: A \sim \alpha \nvdash \alpha\}
$$

It follows from Theorem 3.9 that $R$ satisfies retractability of logical equivalents. Furthermore, from Theorem 3.9 it follows that:

- If $\sim$ satisfies success propagation, then $R$ satisfies non-retractability propagation;

- If $\sim$ satisfies conjunctive constancy, then $R$ satisfies conjunctive completeness.

From Theorem 3.10 and Observation 2.18 it follows that there exists a basic AGM-generated base contraction $\div$ such that $\sim$ is the shielded base contraction induced by $\div$ and $R$. Furthermore, from Theorem 3.10 (c), if $\sim$ satisfies persistence, then $\div$ and $R$ satisfy condition $(\mathbf{R}-\div)$.

(Left-to-right)

Let $A$ be a belief base, $\div$ be a basic AGM-generated base contraction operator on $A$ and $R \subseteq \mathcal{L}$. Let $\sim$ be such that:

$$
A \sim \alpha= \begin{cases}A \div \alpha & \text { if } \alpha \in R \\ A & \text { otherwise }\end{cases}
$$

From Observation $2.18 \div$ satisfies success, inclusion, vacuity, extensionality and disjunctive elimination. From Observation 2.6 (d) it holds that $\div$ also satisfies failure. Hence, from Theorem 3.8 (a) it follows that $\sim$ satisfies relative success, inclusion, vacuity and disjunctive elimination. Furthermore, it follows from Theorem 3.8 that:

- If $R$ satisfies retractability of logical equivalents, then $\sim$ satisfies extensionality (Theorem 3.8 (d)).

- If $R$ satisfies non-retractability propagation, then $\sim$ satisfies success propagation and extensionality (Theorem $3.8(\mathrm{~g})$ ).

- If $R$ satisfies conjunctive completeness and retractability of logical equivalents, then $\sim$ satisfies conjunctive constancy (Theorem $3.8(\mathrm{~h})$ ).

- If $R$ satisfies non-retractability propagation and conjunctive completeness, then $\sim$ satisfies conjunctive constancy (Theorem $3.8(\mathrm{i})$ ).

- If $R$ and $\div$ satisfy condition $(\mathrm{R}-\div)$, then $\sim$ satisfies persistence and extensionality (Theorem 3.8 (b)).

Proof of Observation 5.1. Proof that P-SPMC $\subset$ SP+CC-SPMC, SP+CC-SPMC $\subset$ CC-SPMC, SP+CC-SPMC $\subset$ SP-SPMC, CC-SPMC $\nsubseteq$ SP-SPMC, SP-SPMC $\nsubseteq$ CC-SPMC, CC-SPMC $\subset$ SPMC and SP-SPMC $\subset$ SPMC:

It follows from Example 4.7 that:

(i) SPMC $\nsubseteq \subset$ CC-SPMC, SP-SPMC $\not \subset$ CC-SPMC and SP-SPMC $\nsubseteq$ SP+CC-SPMC;

(ii) SPMC $\nsubseteq$ SP-SPMC, CC-SPMC $\nsubseteq$ SP-SPMC and CC-SPMC $\nsubseteq$ SP+CC-SPMC;

(iii) $\mathrm{SP}+\mathrm{CC}-\mathrm{SPMC} \nsubseteq \mathrm{P}-\mathrm{SPMC}$.

On the other hand it follows from Corollary 4.6 that SP+CC-SPMC $\subseteq$ CC-SPMC, SP+CC-SPMC $\subseteq$ SP-SPMC, CC-SPMC $\subseteq$ SPMC and SP-SPMC $\subseteq$ SPMC. Furthermore, combining Corollary 4.6 and Observations 2.6, 2.21 and 2.22 (b) we can conclude that $\mathrm{P}-\mathrm{SPMC} \subseteq \mathrm{SP}+\mathrm{CC}-\mathrm{SPMC}$.

Proof that P-SKC $\subset$ SP+CC-SKC, SP+CC-SKC $\subset$ CC-SKC, SP+CC-SKC $\subset$ SP-SKC, CC-SKC $\nsubseteq$ SP-SKC, SP-SKC $\nsubseteq$ CC-SKC, CC-SKC $\subset$ SKC and SP-SKC $\subset$ SKC:

That P-SKC $\subseteq$ SP+CC-SKC follows trivially from Corollary 4.10 and Observations 2.6, 2.21 and 2.22 (b).

That SP+CC-SKC $\subseteq$ CC-SKC, SP+CC-SKC $\subseteq$ SP-SKC, CC-SKC $\subseteq$ SKC and SP-SKC $\subseteq$ SKC follow trivially from Corollary 4.10 To prove that SP+CC-SKC $\nsubseteq$ P-SKC, CC-SKC $\nsubseteq$ SP+CC-SKC, SP-SKC $\nsubseteq$ SP+CC-SKC, SP-SKC $\nsubseteq$ CC-SKC, CC-SKC $\nsubseteq$ SP-SKC, SKC $\nsubseteq$ CC-SKC and SKC $\nsubseteq$ SP-SKC it is enough to consider the shielded contractions presented in Example 4.7, attending to Definition 4.9, Corollary 4.10 and to the fact that every partial meet contraction is a kernel contraction (Observation 2.19).

Proof that P-SSKC $\subset$ SP+CC-SSKC, SP+CC-SSKC $\subset$ CC-SSKC, SP+CC-SSKC $\subset$ SP-SSKC, CC-SSKC $\nsubseteq$ SP-SSKC, SP-SSKC $\nsubseteq$ CC-SSKC, CC-SSKC $\subset$ SSKC and SP-SSKC $\subset$ SSKC:

That P-SSKC $\subseteq \mathrm{SP}+\mathrm{CC}-\mathrm{SSKC}$ follows trivially from Corollary 4.13 and Observations 2.6, 2.21 and 2.22 (b).

That SP+CC-SSKC $\subseteq$ CC-SSKC, SP+CC-SSKC $\subseteq$ SP-SSKC, CC-SSKC $\subseteq$ SSKC and SP-SSKC $\subseteq$ SSKC follow trivially from Corollary 4.13 .

To prove that SP+CC-SSKC $\nsubseteq$ P-SSKC, CC-SSKC $\nsubseteq$ SP+CC-SSKC, SP-SSKC $\nsubseteq$ SP+CC-SSKC, SP-SSKC $\nsubseteq$ CC-SSKC, CC-SSKC $\nsubseteq$ SP-SSKC, SSKC $\nsubseteq$ CC-SSKC and SSKC $\nsubseteq$ SP-SSKC it is enough to consider the shielded contractions presented in Example 4.7 , attending to Definition 4.12, Corollary 4.13 and to the fact that every partial meet contraction is a smooth kernel contraction (Observation 2.19). 
Proof that P-SbAGMC $\subset$ SP+CC-SbAGMC, SP+CC-SbAGMC $\subset$ CC-SbAGMC, SP+CC-SbAGMC $\subset$ SP-SbAGMC, CC-SbAGMC $\nsubseteq$ SP-SbAGMC, SP-SbAGMC $\nsubseteq$ CC-SbAGMC, CC-SbAGMC $\subset$ SbAGMC and SP-SbAGMC $\subset$ SbAGMC:

That P-SbAGMC $\subseteq$ SP+CC-SbAGMC follows trivially from Corollary 4.16 and Observations 2.21 and 2.22 (b).

That $\mathrm{SP}+\mathrm{CC}-\mathrm{SbAGMC} \subseteq \mathrm{CC}-\mathrm{SbAGMC}, \mathrm{SP}+\mathrm{CC}-\mathrm{SbAGMC} \subseteq$ SP-SbAGMC, CC-SbAGMC $\subseteq$ SbAGMC and SP-SbAGMC $\subseteq$ SbAGMC follow trivially from Corollary 4.16 .

To prove that SP+CC-SbAGMC $\nsubseteq$ P-SbAGMC, CC-SbAGMC $\nsubseteq$ SP+CC-SbAGMC, SP-SbAGMC $\nsubseteq$ SP+CC-SbAGMC, SP-SbAGMC $\nsubseteq \mathrm{CC}$-SbAGMC, CC-SbAGMC $\nsubseteq$ SP-SbAGMC, SbAGMC $\nsubseteq$ CC-SbAGMC and SbAGMC $\nsubseteq$ SP-SbAGMC it is enough to consider the shielded contractions presented in Example 4.7, attending to Definition 4.15, Corollary 4.16, Observation 3.4 and to the fact that every partial meet contraction is a basic AGM-generated base contraction (Observation 2.19).

\section{References}

[1] Carlos Alchourrón, Peter Gärdenfors, David Makinson, On the logic of theory change: partial meet contraction and revision functions, J. Symb. Log. 50 (1985) 510-530.

[2] Carlos Alchourrón, David Makinson, Hierarchies of regulations and their logic, in: Risto Hilpinen (Ed.), New Studies in Deontic Logic: Norms, Actions, and the Foundations of Ethics, 1981, pp. 125-148.

[3] Carlos Alchourrón, David Makinson, On the logic of theory change: safe contraction, Stud. Log. 44 (1985) $405-422$.

[4] Richard Booth, Eduardo Fermé, Sébastien Konieczny, Ramón Pino Pérez, Credibility-limited revision operators in propositional logic, in: 13th International Conference on the Principles of Knowledge Representation and Reasoning, KR 2012, 2012, pp. $116-125$.

[5] Eduardo Fermé, Sven Ove Hansson, Selective revision, Stud. Log. 63 (3) (1999) 331-342.

[6] Eduardo Fermé, Sven Ove Hansson, Shielded contraction, in: H. Rott, M-A. Williams (Eds.), Frontiers in Belief Revision, in: Applied Logic Series, Kluwer Academic Publishers, 2001, pp. 85-107.

[7] Eduardo Fermé, Sven Ove Hansson, AGM 25 years: twenty-five years of research in belief change, J. Philos. Log. 40 (2011) $295-331$.

[8] Eduardo Fermé, Sven Ove Hansson, Belief Change: Introduction and Overview, Springer Briefs in Computer Science Series, Springer, 2018.

[9] Eduardo Fermé, Martín Krevneris, Maurício Reis, An axiomatic characterization of ensconcement-based contraction, J. Log. Comput. 18 (5) (2008) 739-753.

[10] Eduardo Fermé, Juan Mikalef, Jorge Taboada, Credibility-limited functions for belief bases, J. Log. Comput. 13 (1) (2003) $99-110$.

[11] André Fuhrmann, Sven Ove Hansson, A survey of multiple contraction, J. Log. Lang. Inf. 3 (1994) 39-74.

[12] Peter Gärdenfors, Knowledge in Flux: Modeling the Dynamics of Epistemic States, The MIT Press, Cambridge, 1988.

[13] Peter Gärdenfors, David Makinson, Revisions of knowledge systems using epistemic entrenchment, in: Moshe Y. Vardi (Ed.), Proceedings of the Second Conference on Theoretical Aspects of Reasoning About Knowledge, Los Altos, 1988, Morgan Kaufmann, 1988, pp. 83-95.

[14] Peter Gärdenfors, Hans Rott, Belief revision, in: Dov M. Gabbay, C.J. Hogger, J.A. Robinson (Eds.), Handbook of Logic in Artificial Intelligence and Logic Programming, vol. 4, Oxford University Press, Oxford, UK, 1995, pp. 35-132.

[15] Adam Grove, Two modellings for theory change, J. Philos. Log. 17 (1988) 157-170.

[16] Sven Ove Hansson, New operators for theory change, Theoria 55 (1989) 114-132.

[17] Sven Ove Hansson, Belief Base Dynamics, PhD thesis, Uppsala University, 1991.

[18] Sven Ove Hansson, Belief contraction without recovery, Stud. Log. 50 (1991) 251-260.

[19] Sven Ove Hansson, A dyadic representation of belief, in: Peter Gärdenfors (Ed.), Belief Revision, in: Cambridge Tracts in Theoretical Computer Science, vol. 29, Cambridge University Press, 1992, pp. 89-121.

[20] Sven Ove Hansson, Reversing the Levi identity, J. Philos. Log. 22 (1993) 637-669.

[21] Sven Ove Hansson, Kernel contraction, J. Symb. Log. 59 (1994) 845-859.

[22] Sven Ove Hansson, Semi-revision, J. Appl. Non-Class. Log. 7 (1-2) (1997) 151-175.

[23] Sven Ove Hansson, A survey of non-prioritized belief revision, Erkenntnis 50 (1999) 413-427.

[24] Sven Ove Hansson, A Textbook of Belief Dynamics. Theory Change and Database Updating, Applied Logic Series, Kluwer Academic Publishers, Dordrecht, 1999.

[25] Sven Ove Hansson, Eduardo Fermé, John Cantwell, Marcelo Falappa, Credibility-limited revision, J. Symb. Log. 66 (4) (2001) $1581-1596$.

[26] David Makinson, Screened revision, Theoria 63 (1997) 14-23.

[27] Hans Rott, Preferential belief change using generalized epistemic entrenchment, J. Log. Lang. Inf. 1 (1992) $45-78$.

[28] Hans Rott, Sven Ove Hansson, Safe contraction revisited, in: Sven Ove Hansson (Ed.), David Makinson on Classical Methods for Non-Classical Problems, in: Outstanding Contributions to Logic, vol. 3, Springer, Netherlands, 2014, pp. 35-70.

[29] Mary-Anne Williams, On the logic of theory base change, in: C. MacNish, D. Pearce, L.M. Pereira (Eds.), Logics in Artificial Intelligence. JELIA 1994. Lecture Notes in Computer Science (Lecture Notes in Artificial Intelligence), vol. 838, Springer, Berlin, Heidelberg, 1994, pp. 86-105. 